ACCEPTED BY APJ

Preprint typeset using $\mathrm{LAT}_{\mathrm{E} X} \mathrm{X}$ style emulateapj v. 11/12/01

\title{
ARECIBO MULTI-EPOCH HI ABSORPTION MEASUREMENTS AGAINST PULSARS: TINY-SCALE ATOMIC STRUCTURE
}

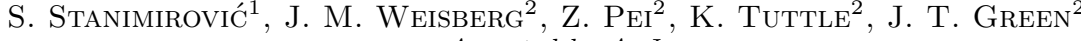 \\ Accepted by ApJ
}

\begin{abstract}
We present results from multi-epoch neutral hydrogen (HI) absorption observations of six bright pulsars with the Arecibo telescope. Moving through the interstellar medium (ISM) with transverse velocities of 10-150 AU yr ${ }^{-1}$, these pulsars have swept across 1-200 AU over the course of our experiment, allowing us to probe the existence and properties of the tiny scale atomic structure (TSAS) in the cold neutral medium (CNM). While most of the observed pulsars show no significant change in their HI absorption spectra, we have identified at least two clear TSAS-induced opacity variations in the direction of B1929+10. These observations require strong spatial inhomogeneities in either the TSAS clouds' physical properties themselves or else in the clouds' galactic distribution. While TSAS is occasionally detected on spatial scales down to $10 \mathrm{AU}$, it is too rare to be characterized by a spectrum of turbulent CNM fluctuations on scales of $10^{1-3} \mathrm{AU}$, as previously suggested by some work. In the direction of B1929+10, an apparent correlation between TSAS and interstellar clouds inside the warm Local Bubble (LB) indicates that TSAS may be tracing the fragmentation of the LB wall via hydrodynamic instabilities. While similar fragmentation events occur frequently throughout the ISM, the warm medium surrounding these cold cloudlets induces a natural selection effect wherein small TSAS clouds evaporate quickly and are rare, while large clouds survive longer and become a general property of the ISM.
\end{abstract}

Subject headings: ISM: clouds — ISM: structure — line: profiles

\section{INTRODUCTION}

For many years, observations of the diffuse interstellar medium (ISM) have traced a whole hierarchy of structures on spatial scales $\gtrsim 1$ pc (cf. Dickey \& Lockman (1990)), often attributed to the ISM turbulence. However, the extremely small-scale end of this spectrum, reaching down to scales of tens to hundreds of AUs, has been largely unexplored. As the kinetic energy cascades from larger to smaller scales, it is expected that the turbulent spectrum will reach its end at so-called "dissipation scales." Understanding of the dissipative properties of turbulence, such as its scale, efficiency, dissipation rate, and ubiquity, are important since the delicate balance between energy injection and dissipation has a profound impact on the stellar - ISM recycling chain. However, many questions still await both theoretical understanding and observational constraints. For example, how is the balance between turbulent energy injection and dissipation maintained? What is the efficiency of the dissipation process? Does it vary with time and galactocentric radius? Is dissipation located only in pockets of "active turbulence", or it is ubiquitous? How do dissipative properties vary with galactic environments?

Starting in 1976 (Dieter et al. 1976), structure in the diffuse ISM has been observed sporadically on scales down to $10^{1-2} \mathrm{AU}$. These findings caused much controversy as the observationally inferred properties were not in accord with the standard ISM picture. With a typical, observationally inferred, HI volume density of $\sim 10^{4} \mathrm{~cm}^{-3}$, and the thermal pressure of $\sim 10^{6} \mathrm{~cm}^{-3} \mathrm{~K}$ (assuming temperature of $\sim 40-70 \mathrm{~K}$ ), the AU-scale "tiny" features appeared significantly over-dense and over-pressured compared with the traditional cold neutral medium (CNM) clouds.

Since the initial observations, the AU-sized struc- tures came to be rather frequently detected, with claims that up to $15 \%$ of the CNM could be in this form (Frail et al. 1994). There has been considerable progress in recent years in understanding of the ISM turbulence and the small-scale structure in the ISM, from both theoretical and observational perspectives [for reviews see Elmegreen \& Scalo (2004); Scalo \& Elmegreen (2004)]. Nevertheless, it remains unclear whether the observed AU-scale features represent the dissipation scales of the $z 1 \mathrm{pc}$ spatial scale turbulence. At the same time, the observational picture has become even more complicated with improved telescope sensitivity, resulting in the detection of new populations of possibly related sub-parsec ISM clouds (Braun \& Kanekar 2005; Stanimirović \& Heiles 2005; Begum et al. 2010). The "low column density clouds" observed by Stanimirovic \& Heiles (2005) have a peak optical depth of only $10^{-3}$ to $10^{-2}$ and an inferred size of 500-5000 AU.

Motivated by the recent theoretical and observational efforts in understanding the nature and origin of the tinyscale ISM structure, we have undertaken multi-epoch observations of the HI absorption against a set of bright pulsars. Because of pulsars' relatively high proper motion and transverse speeds (typically $\sim 10-10^{2} \mathrm{AU} \mathrm{yr}^{-1}$ ), pulsar HI absorption profiles obtained at different epochs sample CNM structure on AU spatial scales. In addition, the pulsed nature of pulsars' emission allows spectra to be obtained on and off source without moving the telescope, therefore sampling both emission and absorption along almost exactly the same line of sight. Stanimirović et al. (2003) summarized preliminary results from this project for three pulsars, B0823+26, B1133+16 and B2016+28. Here we present results for all pulsars with full analysis.

${ }^{1}$ Department of Astronomy, University of Wisconsin, Madison, WI 53706; sstanimi@astro.wisc.edu

2 Department of Physics and Astronomy, Carleton College, Northfield, MN 55057 
The structure of this paper is organized in the following way. Section 2 provides a short summary of the main observational and theoretical results of the AU-scale structure in neutral gas. In Section 3 we summarize our observing and data processing strategies. Results from our investigation of changes in absorption profiles' integrated optical depth properties (c.f. equivalent widths) are presented in Section 4. while in Section 5 we search for HI absorption feature optical depth variability. To facilitate further data analysis we derive the spin temperature and HI column density in the direction of all pulsars in Section 6. In Section 7, we investigate in detail the properties of the ISM along lines of sight toward our pulsars. In Section 8 we study the properties of the most significant AU-scale features found in this study. We discuss our results and compare them with several theoretical models in Section 9, and summarize our findings in Section 10.

\section{BACKGROUND}

While structure on AU scales has been observed in neutral, ionized, and molecular flavors of diffuse gas, it is still not clear whether all these features trace a single or multiple phenomena. Our work focuses only on the tiny-scale atomic structure (TSAS; Heiles 1997) and we summarize results from two different observational approaches used to study TSAS in the CNM below in 2.1 and 2.2 as well as several theoretical considerations of the TSAS phenomenon in 2.3 In addition to observational methods discussed here, TSAS in the CNM has been also observed through temporal (probing scales of $\sim 1$ to tens of AUs) and spatial (probing scales of a few thousands of AU) variability of optical absorption lines (Mever \& Blades 1996; Andrews et al. 2001; Crawford et al. 2000; Lauroesch \& Mever 2003). The reader is referred to excellent articles in the ASP Conf. Series Vol. 365 (Haverkorn \& Goss 2007) for complementary details on optical absorption line studies, or the Galactic tiny-scale structure in the ionized and molecular gas. Finally, there are also some indications that even damped Lyman $\alpha$ absorbing systems at high redshift may contain small-scale HI structure on scales of $\sim 10-100 \mathrm{AU}$ (Kanekar \& Chengalur 2001).

\subsection{Observations of TSAS via spatial mapping of HI absorption line profiles across extended background} sources

Single baseline very long baseline interferometric (VLBI) observations of the quasar 3C147 by Dieter et al. (1976) were the first to infer the existence of a tiny-scale atomic cloud, having a size of $70 \mathrm{AU}$ and $\mathrm{HI}$ volume density of $10^{5} \mathrm{~cm}^{-3}$, based on variations in absorption line profiles in the directions of separate source components. Subsequent VLBI observations by Diamond et al. (1989) found evidence for 25-AU HI clouds in directions toward 3C138, 3C380, and 3C147. Particularly large variations were noticed in the case of $3 \mathrm{C} 138$.

The first images of $\mathrm{HI}$ optical depth distribution in the direction of extragalactic sources were obtained by Davis et al. (1996) toward 3C138 and 3C147, using the MERLIN array and the European VLBI Network. Faison et al. (1998) and Faison \& Goss (2001) used the Very Long Baseline Array (VLBA) to image HI absorption toward seven sources. Significant variations in HI optical depth with $\Delta \tau$ of 0.1 and $\sim 1$, respectively, were found toward only two sources, 3C147 and 3C138.

The latest results on $3 \mathrm{C} 138$ by Brogan et al. (2005), in a three-epoch series of observations, show clear evidence for spatial variations of $\Delta \tau \sim 0.5$ on scales of $25 \mathrm{AU}$. In addition, temporal changes in HI optical depth have been found over a period of 7 years, with implied transverse velocities of order $20 \mathrm{~km} \mathrm{~s}^{-1}$ (see Brogan et al. (2007) for a thorough review of these measurements). Some $10 \%$ of pixels in their optical depth images have $\Delta \tau>5-\sigma$. Assuming that most of the CNM along the line of sight contains small-scale structure, they estimated the volume filling factor of about $1 \%$. They proposed that TSAS is ubiquitous, and that the lower measured levels of variations in the other cases result from a selection effect due to incomplete sampling of the relevant angular and spatial scales.

Recently, Goss et al. (2008) used MERLIN observations of 3C161 and found $\Delta \tau \sim 1.0-1.5$ on scales of $\sim 500 \mathrm{AU}$. A slightly smaller level of variations (typically $\Delta \tau \sim 0.2$ ) was found in the case of $3 \mathrm{C} 147$, on scales of about $10 \mathrm{AU}$ (Lazio et al. 2009).

\subsection{Observations of TSAS via time variability of HI absorption profiles against pulsars}

In the late 1980s, sufficiently accurate and repeated measurements of $\mathrm{HI}$ absorption profiles against pulsars began to be made, and it was noticed that pulsar ISM spectra changed over time in some cases, suggesting inhomogeneities in the intervening gas. For example, Clifton et al. (1988) found that the HI absorption spectrum of PSR B1821+05 changed significantly between 1981 and 1988, with the appearance at the latter epoch of a previously unobserved feature with $\tau \sim 2$ and $\Delta v \sim 1 \mathrm{~km} \mathrm{~s}^{-1}$. Deshpande et al. (1992) showed that between $\sim 1976$ and 1981, HI absorption toward B1154-62 did not change significantly; while toward B1557-50, a variation with $\Delta \tau \sim 1$ was interpreted as a cloud of size in the 1000 AU range.

The early pulsar HI results inspired Frail et al. (1994) to undertake a dedicated multi-epoch pulsar HI absorption experiment at Arecibo. Six pulsars were observed at three epochs, with time baselines ranging from $0.7-1.7$ yr. These authors reported the presence of pervasive variations with $\Delta \tau \sim 0.03-0.7$, and associated HI column densities of $10^{19}-5 \times 10^{20} \mathrm{~cm}^{-2}$; on scales of 5-100 AU. They indicated that $10-15 \%$ of cold HI is in the tiny structures, and additionally detected a correlation between absorption equivalent width variations and equivalent width itself. These results appeared to buttress the earlier VLBI findings of TSAS, and they provided a strong impetus for further experimental and theoretical work.

The recent era of pulsar TSAS experiments began with the Parkes observations of Johnston et al. (2003). Surprisingly, these investigators found no significant optical depth variations in their three-epoch, 2.5-yr observations of three pulsars, and were able to place an upper limit on column density variations toward PSR B1641-45 of $10^{19}$ $\mathrm{cm}^{-2}$, significantly below the Frail et al. (1994) detections. They showed that the earlier experiment did not fully account for the large increases in noise in absorption spectra at the line frequency, so that some of the apparently 
significant variations actually were not. While no significant variations were seen by Johnston et al. (2003) during the 2.5 years of their TSAS experiment, they detected variations in the spectrum of PSR B1557-50 when compared with measurements made five years earlier. This is the same pulsar whose spectrum was noted earlier by Deshpande et al. (1992) to vary on similar timescales in the late $1970 \mathrm{~s}$. In combining the results from four measurements over twenty-five years, Johnston et al. (2003) concluded that the cloud causing the variations is $\sim 1000$ AU in size, with a density of $\sim 10^{4} \mathrm{~cm}^{-3}$.

Minter et al. (2005) performed a particularly exhaustive TSAS study on PSR B0329+54, which is very bright and almost circumpolar at the Green Bank Telescope. The investigators observed the pulsar continuously for up to 20 $\mathrm{hr}$ in eighteen observing sessions over a period of $1.3 \mathrm{yr}$. They detected no HI optical depth variations $(\Delta \tau<0.026$ in most cases) for pulsar transverse offsets ranging from 0.005-25 AU.

We and our colleagues published early results from a new set of multi-epoch Arecibo observations (Stanimirović et al. 2003). Based on non-detections of TSAS in the direction of three pulsars $(\mathrm{B} 0823+26$, $\mathrm{B} 1133+16$, and B2016+28), we suggested that TSAS is not ubiquitous in the ISM and could be related to isolated events. The current work elaborates these themes.

\subsection{Theoretical considerations of TSAS}

Several explanations were proposed to reconcile the observations of TSAS with theory. Heiles (1997) proposed that TSAS features could be curved filaments and/or sheets that happen to be aligned along our line-of-sight. By combining low temperature and a geometrical elongation, the apparently problematic volume density and thermal pressure can be significantly reduced. We discuss this model in some detail in Section 9.1 .

Deshpande (2000) suggested that TSAS represents the tail-end of the turbulent ISM spectrum which exists on larger spatial scales, and showed that the observed optical depth differences are consistent with a single powerlaw description of the HI optical depth distribution as a function of spatial scale. It was also pointed out that the over-density of TSAS is due to a misinterpretation of observations: the observed variations in optical depth sample the square-root of the structure function of the HI optical depth, not directly the power spectrum of optical depth fluctuations. If the power spectrum of the HI optical depth distribution in the direction of Cas $\mathrm{A}$, with a measured slope of 2.75 (Deshpande et al. 2000) over a range of spatial scales from 0.02 to $4 \mathrm{pc}$, is extrapolated to AU scales then $\Delta \tau=0.2-0.4$ is expected on scales of 50-100 AU. We note that this slope was confirmed recently in an independent experiment by Roy et al. (2010).

Gwinn (2001) proposed that optical depth fluctuations seen in multi-epoch pulsar observations are a scintillation phenomenon combined with a velocity gradient across the absorbing HI. While in the case of Deshpande (2000) optical depth variations are expected to increase with the size of structure, the scintillation phenomenon predicts maximum variations on the very small spatial scales probed by interstellar scintillation. However, dedicated observations of PSR J1456-6843 by Gwinn et al. (2007) failed to detect any scintillation effects on the $\mathrm{HI}$ absorption line.

One of the main problems regarding TSAS is its overpressure relative to the traditional thermal pressure of the ISM, which suggests that TSAS can not persist nor be pervasive. However, recent numerical simulations of the dynamic and turbulent ISM find substantial departures from pressure equilibrium. Instead of welldefined thermal phases, gas with a wide range of densities and temperatures is found in simulations (Mac Low et al. 2005; Hennebelle \& Audit 2007). Such results are supported by observations of the CI fine-structure lines by Jenkins \& Tripp (2001) who found evidence for the existence of over-pressured gas $\left(P / k \gtrsim 10^{5} \mathrm{~cm}^{-3} \mathrm{~K}\right)$. While these studies suggest that high-pressure TSAS could be a natural product of the highly dynamic ISM, the fraction of high-pressure gas in the ISM, as well as the physical processes responsible for their formation, vary hugely across simulations. The only way to constrain TSAS properties and abundance is through sensitive observations.

\section{OBSERVATIONS AND DATA PROCESSING}

\subsection{The observing and basic data processing procedure}

We used the Arecibo telescop $3^{3}$ and the Caltech Baseband Recorder (CBR) backend (Jenet et al. 1997), to measure HI absorption spectra of six pulsars at multiple epochs. We chose to observe the same sources as Frail et al. (1994) in order to enhance the number of available time baselines for comparison. Table 1 lists several basic parameters for our targets: Galactic coordinates $(l / b)$, the dispersion measure (DM), continuum equivalent flux density at $20 \mathrm{~cm}\left(\mathrm{~S}_{20}\right)$, distance $(\mathrm{D})$, the transverse velocity $\left(\mathrm{V}_{t}\right)$, the transverse distance traveled in a year $\left(\mathrm{L}_{\perp}\right)$, and the pulsar-on antenna temperature $\left(T_{\mathrm{PSR}}\right)$. The tabulated flux density and $T_{\text {PSR }}$ represent average values obtained from long integrations, whereas the actual pulsar flux density and $T_{\mathrm{PSR}}$ vary on timescales from several minutes to several hours due to interstellar scintillation. Distances for all six pulsars (column 5) were determined from interferometric parallax measurements. The pulsar-on antenna temperature $T_{\mathrm{PSR}}=G S_{20} P / W_{50}$, with the L-band gain $G=7.5 \times 10^{-3} \mathrm{~K} \mathrm{mJy}^{-1}$ (Heiles et al. 2001), and pulsar period $P$ and $50 \%$ pulse width $W_{50}$ from the ATNF pulsar Catalogue (Manchester et al. 2005).

The observing and basic data processing procedures were described in Stanimirović et al. (2003) and Weisberg et al. (2008). Relative to the previous large multi-epoch experiment by Frail et al. (1994), our fourlevel spectrometer is less prone to systematic errors when observing these strong and highly-variable sources. We had four observing sessions, referred to below as S1, S2, S3, S4; for epochs 2000.62, 2000.95, 2001.70, and 2001.86, respectively. The typical integration time per pulsar was about 8 hours for each of four sessions.

The raw complex voltage samples recorded with the CBR were processed at Caltech's Center for Advanced Computation and Research to obtain a data cube of pulsar intensity as a function of pulsar rotational phase and radio frequency. For each scan, portions of the data cube during

3 The Arecibo Observatory is part of the National Astronomy and Ionosphere Center, operated by Cornell University under a cooperative agreement with the National Science Foundation. 

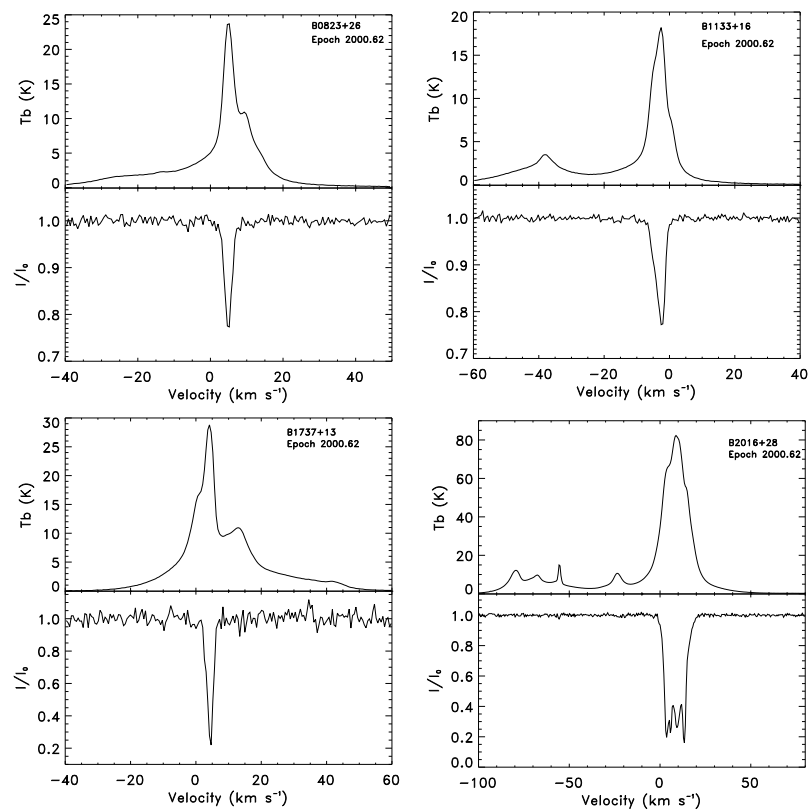

Fig. 1.- Emission (top) and absorption (bottom) profiles in the direction of B0823+26, B1133+16, B1737+13, and B2016+28 obtained at the first epoch (August 2000). The emission spectra were scaled to match the brightness temperature from the Leiden-Dwingeloo survey.
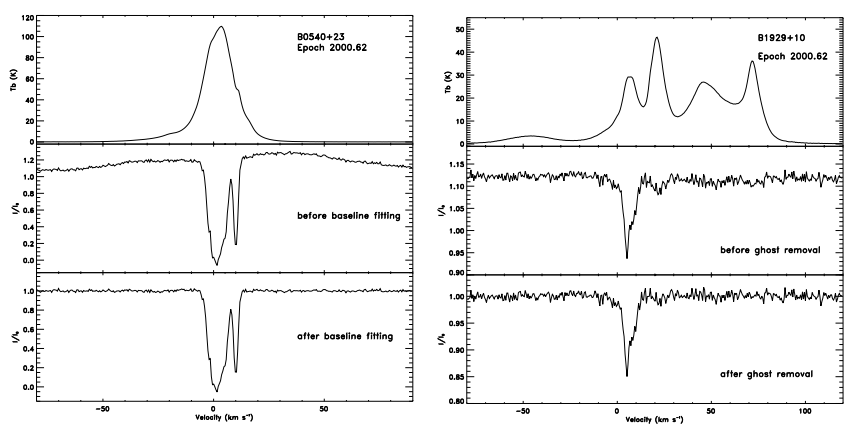

FIG. 2.- Emission and absorption profiles in the direction of B0540+23 and B1929+10, obtained at the first epoch (August 2000). The middle panels show the absorption profiles before baseline fitting for B0540+23, and before ghost removal (see 3.2 for B1929+10. In the case of B0540+23, a large ripple is obvious in the middle panel, caused by the interstellar scintillation. For B1929+10, a faint inverted copy of the $\mathrm{H}$ I emission spectrum is noticeable especially around $20 \mathrm{~km} \mathrm{~s}^{-1}$. The bottom panels show the absorption spectra after baseline and ghost correction, respectively.

TABLE 1

BASIC PARAMETERS OF PULSARS OBSERVED IN THIS STUdy, TABULATED OR DERIVED USING THE REFERENCE GIVEN IN THE LAST Column and the ATNF Pulsar Catalogue (Manchester et AL. 2005).

\begin{tabular}{ccccccccc}
\hline \hline $\begin{array}{c}\text { PSR } \\
(\mathrm{B} 1950)\end{array}$ & $\begin{array}{c}l / b \\
\mathrm{deg}\end{array}$ & $\begin{array}{c}\mathrm{DM} \\
\left(\mathrm{pc} \mathrm{cm}^{-3}\right)\end{array}$ & $\begin{array}{c}\mathrm{S}_{20} \\
(\mathrm{mJy})\end{array}$ & $\begin{array}{c}\mathrm{D} \\
(\mathrm{kpc})\end{array}$ & $\begin{array}{c}\mathrm{V}_{\mathrm{t}} \\
\left(\mathrm{km} \mathrm{s}^{-1}\right)\end{array}$ & $\begin{array}{c}\mathrm{L}_{\perp} \\
\left(\mathrm{AU} \mathrm{yr}^{-1}\right)\end{array}$ & $\begin{array}{c}T_{\mathrm{PSR}} \\
(\mathrm{K})\end{array}$ & Reference \\
\hline B0540+23 & $184.36 /-3.32$ & 77.7 & 9 & 3.5 & 377 & 80 & 2.7 & Harrison et al. (1993) \\
$\mathrm{B} 0823+26$ & $196.96 / 31.74$ & 19.5 & 10 & 0.4 & 192 & 39 & 6.9 & Gwinn et al. (1986) \\
$\mathrm{B} 1133+16$ & $241.90 / 69.19$ & 4.8 & 32 & 0.4 & 631 & 135 & 9.0 & Brisken et al. $(2002)$ \\
$\mathrm{B} 1737+13$ & $37.08 / 21.68$ & 48.9 & 3.9 & 4.7 & 672 & 142 & 0.7 & Brisken et al. $(2003)$ \\
$\mathrm{B} 1929+10$ & $47.38 /-3.88$ & 3.2 & 36 & 0.3 & 177 & 37 & 8.3 & Chatteriee et al. (2004) \\
$\mathrm{B} 2016+28$ & $68.10 /-3.98$ & 14.2 & 30 & 1.0 & 30 & 7 & 8.4 & Brisken et al. (2002) \\
\hline
\end{tabular}


and off the pulsar pulse were accumulated to obtain the 'pulsar-on' and 'pulsar-off' spectra. The pulsar absorption spectrum represents the 'pulsar-on' - 'pulsar-off' spectrum for each scan, whose baseline was then flattened by performing frequency switching and normalizing by the offline pulsar intensity. In accumulating pulsar absorption spectra from different $\sim 2-$ min scans, each spectrum was given a weight proportional to $T_{\mathrm{PSR}}^{2}$, where $T_{\mathrm{PSR}}$ is the antenna temperature of the pulsar.

The frequency-switched 'pulsar-off' spectra correspond to the $\mathrm{H}$ I emission spectra in pulsar directions. As no temperature calibration was performed during the observations, we have scaled these spectra to match the $\mathrm{H}$ I brightness temperature from the Leiden-Dwingeloo survey (Burton \& Hartmann 1994). Final absorption and emission spectra for all pulsars, from the first observing epoch (2000.62), are shown in Figures 1 and 2. Our spectrometer, consisting of 4096 frequency channels across $10 \mathrm{MHz}$ total bandwidth, has a velocity channel spacing of $0.52-$ $\mathrm{km} \mathrm{s}^{-1}$. However, adjacent spectral channels are not independent, which can lead to distortion of narrowband signals such as the ones we are studying (Rohlfs \& Wilson 2004). To ameliorate this problem, we Hanning smoothed all processed absorption spectra, yielding a final velocity resolution of $1.04 \mathrm{~km} \mathrm{~s}^{-1}$. Note that Hanning smoothing was not applied in our preliminary analyses [Stanimirovic et al. (2003); Weisberg \& Stanimirovic (2007)].

\subsection{Baseline fitting of absorption spectra}

To flatten the baseline of the final absorption spectra we fitted a polynomial function to the unabsorbed portions of the spectrum. For pulsars B0823+26, B1133+16, $\mathrm{B} 1737+13$ and $\mathrm{B} 2016+28$ generally a 1 st or 2 nd order polynomial function was fitted and then the full spectrum was divided by the fitted function. A higher order polynomial was required for the $3 \mathrm{rd}$ session data for B2016+28 due to ripples in the baseline structure, most likely cased by interstellar scintillation.

PSR B0540+23 data were heavily affected by interstellar scintillation. This pulsar's scintillation bandwidth at 1.4 $\mathrm{GHz}$ is $0.3 \mathrm{MHz}$ (Gwinn 2001), which resulted in numerous ripples across our band (see Figure 2, left middle panel). We needed to fit and divide by a 15th order polynomial to flatten the baseline for this pulsar (see bottom panel in Figure 2). However, as emphasized in Section 4.1, we do not use this pulsar's data for our analysis due to large scintillation effects which presumably led to the unphysically $\left(I / I_{0}<0\right)$ deep absorption features. Noise and calibration errors in the pulsar absorption spectrum are magnified by a factor of $e^{\tau}$ when the absorption spectrum is expressed in terms of the ultimately desired optical depth $\tau$. With this pulsar's optical depth near infinity, these errors become so large as to render our measurements unusable.

B1929+10 data, as shown in Figure 2 (right, middle panel), suffer from the 'ghost effect' (Weisberg et al. 1980). The main absorption feature is at $5 \mathrm{~km} \mathrm{~s}^{-1}$. However several additional features are visible from 20 to 80 $\mathrm{km} \mathrm{s}^{-1}$ which represent a false image of the pulsar-on emission spectrum. The usual reason for the ghosts is an incorrect scaling of total power received by the spectrometer in the case of strong and variable sources. As the ghost is a faint copy of the pulsar-on emission spectrum in the pulsar absorption spectrum, we can exorcize the ghost by fitting for and removing the former from the latter.

To do so we have performed a least-squares fit of the $I(v) / I_{0}$ absorption spectrum to a ghost of fitted amplitude $A$, and a fitted linear baseline slope $B$ plus constant $C$, all to spectral channels lacking true absorption:

$$
I(v) / I_{0}=A T_{\mathrm{PSR}-\text { on }}(v)+B v+C,
$$

where $T_{\mathrm{PSR}-\mathrm{on}}(v)$ is the measured pulsar-on spectrum. The exorcized absorption spectrum is then:

$$
\left(I(v) / I_{0}\right)_{\text {corrected }}=\left[I(v) / I_{0}-A T_{\mathrm{PSR}-\mathrm{on}}(v)-B v\right] / C \text {. }
$$

The exorcized PSR B1929+10 absorption spectrum is shown in Figure 2, right bottom panel. The fitted parameters are listed in Table 2 .

\subsection{Noise envelopes}

For each absorption spectrum we have calculated an expected noise envelope which will be used to assess the significance of any observed temporal variations. The expected noise level $\Delta T(v)$ is proportional to the overall system temperature $T_{\text {sys }}(v)$, which has the following contributions. (1) The receiver temperature $T_{\mathrm{rx}} \sim 25-30 \mathrm{~K}$. (2) The sky background, which is typically of order of $1-2 \mathrm{~K}$, based on the all-sky survey at $408 \mathrm{MHz}$ by Haslam et al. (1982) and after scaling to $1.4 \mathrm{GHz}$ using a spectral index of -2.6 . (3) The contribution from the HI emission which can be very significant and which varies with velocity. For example the rms noise on-line is three times higher than the rms noise off-line for the case of B0540+23. (4) At Arecibo, the pulsar continuum emission itself is also sometimes sufficiently strong to provide the principal contribution to the system temperature.

Since an observed pulsar-on spectrum is identically the sum of these four contributions, the expected noise in the pulsar-on spectrum should be proportional to the pulsaron spectrum. Furthermore, owing to pulsar-off integration times being $\sim 10-100$ times longer than pulsar-on integration times, the pulsar-off spectrum adds negligible additional noise to the absorption spectrum. Hence our expected noise envelopes (shown in Figures 510) are generated by scaling the observed pulsar-on spectra by a multiplicative factor that matches them to the observed noise in the off-line portions of the absorption spectra. We have then propagated uncertainties associated with baseline fitting and ghost removal procedures to derive the final expected noise envelopes. To estimate significance envelopes for difference spectra shown in Section 5 (Figures 3-8) or for the equivalent width analysis in Section 4 , appropriate noise envelopes for individual absorption profiles were added in quadrature.

To investigate the properties of noise in emission-free channels, we have plotted the histogram of data points located off-line for each difference spectrum (not shown in the paper). Most histograms are consistent with Gaussian statistics with a standard deviation in agreement or close to our derived 1- $\sigma$ noise level. In several cases there is a small discrepancy between the measured off-line noise and derived 1- $\sigma$ noise envelopes. This is not surprising after the propagation of errors due to baseline, and especially ghost, fitting. For at least three of our pulsars we have achieved excellent sensitivity. We discuss the rms on-line noise level of the final absorption spectra in Section 5. 
TABLE 2

Fitted parameters for the 'Ghost' Excision in the CASE of B1929+10 (SECtion 3.2).

\begin{tabular}{cccc}
\hline \hline Session & $\begin{array}{c}A \\
\left(\mathrm{~K}^{-1}\right)\end{array}$ & $\begin{array}{c}B \\
\left(10^{-5} \mathrm{~km}^{-1} \mathrm{~s}\right)\end{array}$ & $C$ \\
\hline 1 & $-4.3 \pm 0.4$ & $-3.0 \pm 0.6$ & $1.148 \pm 0.003$ \\
2 & $-9.6 \pm 0.4$ & $6.1 \pm 0.3$ & $1.147 \pm 0.002$ \\
3 & $-7.5 \pm 0.4$ & $4.8 \pm 0.3$ & $1.128 \pm 0.002$ \\
4 & $-14.9 \pm 0.7$ & $-0.3 \pm 0.3$ & $1.144 \pm 0.002$ \\
\hline
\end{tabular}

\section{EQUIVALENT WIDTH VARIATIONS}

Our main goal is to search for variations in the HI absorption profiles obtained at different observing epochs. We have employed two methods: (i) in this section we look for changes in integrated properties such as the equivalent width, and (ii) in Section [5] we investigate spectra differenced between each pair of observing epochs.

We have measured the equivalent width

$$
\mathrm{EW}=\int \tau d v=-\int \ln \left(I / I_{0}\right) d v
$$

in units of $\mathrm{km} \mathrm{s}^{-1}$, for each observing epoch. The optical depth profiles were integrated over the 'on-line' velocity regions. To avoid low signal/noise parts of absorption spectra, as well as line wings which may be affected by small baseline imperfections, we have defined the 'on-line' regions as roughly encompassing velocities with $\tau(v)>2-\sigma$. For each pulsar a single region was used for all observing epochs: $[-10,15] \mathrm{km} \mathrm{s}^{-1}$ for B0540+23, $[2,7] \mathrm{km} \mathrm{s}^{-1}$ for B0823+26, $[-7,0] \mathrm{km} \mathrm{s}^{-1}$ for B1133+16, $[2,6] \mathrm{km} \mathrm{s}^{-1}$ for $\mathrm{B} 1737+13,[-3,12] \mathrm{km} \mathrm{s}^{-1}$ for B1929+10 and $[-2,21]$ $\mathrm{km} \mathrm{s}^{-1}$ for $\mathrm{B} 2016+28$. In addition, by averaging optical depth profiles for all epochs we have estimated the mean equivalent width for each pulsar $(\overline{\mathrm{EW}})$.

To compare EW from one epoch to the next we have calculated $\Delta \mathrm{EW}(\Delta t)=\left[\mathrm{EW}\left(t_{1}\right)-\mathrm{EW}\left(t_{2}\right)\right]$. These values, as a function of time baselines $\Delta t$ (bottom axis) or traversed spatial scales $l$ (shown on the top axis), are shown for all pulsars in Figure 3. B0540+23 was excluded from this analysis as its optical depth profile is uncertain around 0 $\mathrm{km} \mathrm{s}^{-1}$. To convert from time intervals $\Delta t$ to spatial scales $l$, we use $l=\Delta t \times L_{\perp}$, where $L_{\perp}$ is provided in Table 1 . Our spatial scales given below should be multiplied by an (unknown) fractional distance factor $f=D_{\mathrm{HI}} / D_{\mathrm{PSR}}$ to account for the fact that absorbing HI is located between us and the pulsar. Note that some authors have arbitrarily fixed $f$; e.g. for Minter et al. (2005) $f=0.5$. (When we assemble various workers' results in this paper, we redefine all such values onto our scale.)

As shown in Figure 3. in the case of B0823+26, $\mathrm{B} 1133+16, \mathrm{~B} 1737+13$ and $\mathrm{B} 2016+28$ all variations in $\Delta \mathrm{EW}$ are essentially within $2-\sigma$ and are most likely not significant. However, in the case of B1929+10, baselines of 0.33 and 1.24 yrs show changes in $\Delta \mathrm{EW}$ at a $3.3-\sigma$ and $3.5-\sigma$ level, respectively. In addition, the $0.16 \mathrm{yr}$ baseline shows a variation at a $2.8-\sigma$ level, while the $0.75 \mathrm{yr}$ baseline shows a 2.5- $\sigma$ change. To quantify the significance of $\Delta \mathrm{EW}$ variability we test the null hypothesis of $\Delta \mathrm{EW}$ being constant with time. We fit a constant $\Delta \mathrm{EW}$ model to measurements in Figure 3 and find $\chi^{2} /$ dof $=0.4(B 0823+26)$,
$0.8(\mathrm{~B} 1133+16), 1.3(\mathrm{~B} 1737+13), 6.0(\mathrm{~B} 1929+10)$, and $1.2(\mathrm{~B} 2016+28)$. We conclude that $\triangle \mathrm{EW}$ changes for B1929+10 can not be fit with a constant model, while for all other pulsars no significant variability of $\Delta \mathrm{EW}$ is observed. This $\triangle \mathrm{EW}$ variability, as further analyzed in Section 7.1, can be interpreted as being due to TSAS.

In Figure 4 we plot $\Delta \mathrm{EW}$, separately for individual pulsars, but as a function of $\overline{\mathrm{EW}}$. This plot can be directly compared with Figure 4 from Frail et al. (1994). Our results for B0823+26, B1133+16 and B1929+10 agree roughly with Frail et al.'s, $|\Delta \mathrm{EW}|<0.1$. However $\Delta \mathrm{EW}$ for B1737+13 and B2016+28 found in Frail et al. (1994) were significantly larger than what we find. A particularly striking difference is the case of B2016+10, where Frail et al. (1994) found $\Delta \mathrm{EW} \sim 1-5$, while we find only $\Delta \mathrm{EW} \sim 0.1-0.3$. We believe that at least some of the variations Frail et al. saw were due to small calibration errors. Looking at our whole sample, the fractional variation $\Delta \mathrm{EW} / \overline{\mathrm{EW}}$ spans a range from $0 \%$ to $11 \%$, with a median of $4 \%$. This is significantly smaller from a median value of $13 \%$ found in Frail et al. (1994).

As a conclusion, we find significant variations in $\Delta \mathrm{EW}$ only for B1929+10, with two largest changes being at a fractional level of $11 \%$. The frequency of variations and the level of variations (as well as upper limits) are significantly smaller than what was found previously for the same sources by Frail et al. (1994). Our results do not rule out the finding by Frail et al. (1994) that $|\Delta \mathrm{EW}|$ increases with EW. However, if this relationship exists, it is at a much smaller level than suggested by Frail et al.

\section{VARIATIONS FROM DIFFERENCE SPECTRA}

Considering that TSAS may have temperature as low as $15 \mathrm{~K}$ (Heiles 1997), the change in absorption profiles may occur over $\lesssim 1 \mathrm{~km} \mathrm{~s}^{-1}$ and appear in only one or two velocity channels in our spectra. Such narrow changes may get washed out when calculating the equivalent width. We therefore in this section compare all pairs of absorption profiles for a given pulsar, and investigate absorption spectrum differences, $\Delta\left(I / I_{0}\right) \sim \Delta \tau$. With four observing epochs we have six difference spectra (Figures [5] to 10). Overlaid atop each difference spectrum is a $\pm 2-\sigma$ significance envelope calculated from noise envelopes discussed in Section 3.3 .

In summary, we see changes in difference spectra at a $3-\sigma$ level only in the case of B1929+10 and only for the two baselines where we also find changes in $\Delta \mathrm{EW}$ at a $>3-\sigma$ level. We note that B1133+16 has one feature above 3$\sigma$ (s4-s3 baseline) at a velocity of $-30 \mathrm{~km} \mathrm{~s}^{-1}$, however this likely comes from RFI as is seen in emission in the 

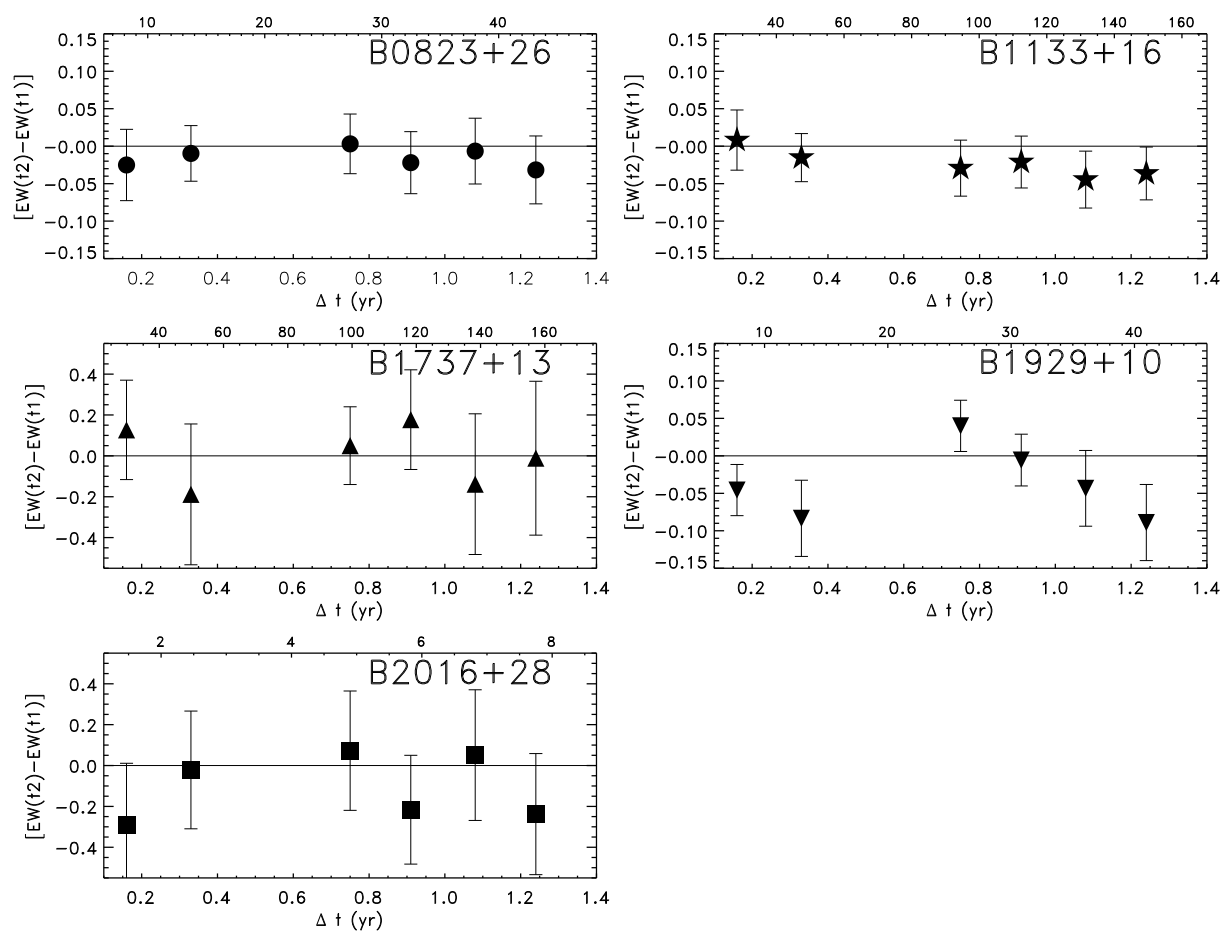

FIG. 3.- Temporal change in the equivalent width between two epochs, $\Delta \mathrm{EW}(\Delta t)=\left[\mathrm{EW}\left(t_{2}\right)-\mathrm{EW}\left(t_{1}\right)\right]$. Plotted error bars represent $\pm 2-\sigma$ uncertainties. The top x-axis on each plot shows the corresponding spatial scales, $l=\Delta t \times L_{\perp}$.

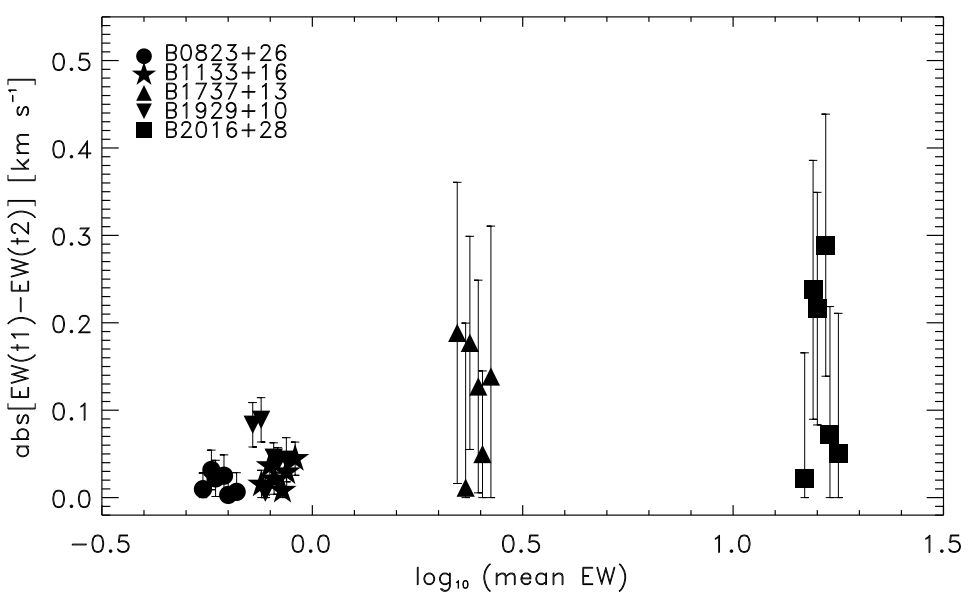

FIG. 4. - The absolute value of $\Delta \mathrm{EW}$ as a function of the mean EW for each pulsar, $\overline{\mathrm{EW}}$. Plotted error bars represent $\pm 1-\sigma$ uncertainties. 
absorption spectrum from session 3. Similarly, B1737+13 has a significant variation at a velocity of $\sim 35 \mathrm{~km} \mathrm{~s}^{-1}$ on 3 baselines, all involving the session 1 absorption spectrum; this corresponds to an (unphysical) emission feature and is most likely due to RFI.

For three of our pulsars (B0823+23, B1133+16 and B1929+10) we have excellent sensitivity for TSAS experiments, $\Delta \tau \sim 0.01-0.02(2-\sigma$ in the on-line portion of the spectrum). Only one recent experiment (Minter at al. 2005) reached similar sensitivity but primarily over smaller spatial scales. For B1737+13 and B2016+28 we have reached 2- $\sigma$ sensitivity of $0.1-0.2$. Although this is slightly higher, several pulsar and VLBA TSAS detections have been claimed at this level. We provide below brief comparison between our observations for individual pulsars and previous TSAS experiments.

\subsection{Difference spectra of individual sources}

\subsubsection{PSR B0540+23}

The corresponding tranverse distances traveled by B0540+23 during the course of our observations are 13, 26, 60, 73, 86 and 100 AUs. Frail et al. (1994) concluded that there were clear signs of significant optical depth variations in some absorption components for this pulsar. In fact both our and Frail et al.'s spectra show $\left(I / I_{0}\right)$ slightly less than zero near $0 \mathrm{~km} \mathrm{~s}^{-1}$ as well as scintillation ripples. As already discussed in Section 3.2, we do not use data for this pulsar for our further analyses and do not show difference spectra.

\subsubsection{PSR B0823+26 (Figure 5)}

With $L_{\perp}=39 \mathrm{AU} \mathrm{yr}^{-1}$, the time intervals covered by our experiment translate to spatial scales of $6,13,29,35$, 42 and 48 AUs. There are no data points with $\Delta \tau$ above 3- $\sigma$ in difference spectra for all six time baselines. We conclude that no significant change in absorption profiles is found down to a $\Delta \tau$ level of 0.03 (a $2-\sigma$ level on $\mathrm{H}$ I line). Frail et al. (1994) detected only marginal changes over 0.6 yr, but reported significant variations of $\Delta \tau \sim 0.07$ in 1.1 yr. We do not confirm this reported variation on any of our temporal baselines although our 2- $\sigma$ sensitivity level on all baselines is at least two times smaller than the level of variations found by Frail et al. (1994). We discussed this further in Stanimirović et al. (2003) and concluded that calibration and noise issues affected the Frail et al. analyses.

\subsubsection{PSR B1133+16 (Figure 6)}

As this pulsar has $L_{\perp}=135 \mathrm{AU} \mathrm{yr}^{-1}$ a wide range of spatial scales is probed by our observations, from $20 \mathrm{AU}$ to $170 \mathrm{AU}$. No variability at $\gtrsim 3-\sigma$ was found and we conclude that no significant variations were detected in the direction of this pulsar down to a $\Delta \tau$ level of about 0.02 . Frail et al. (1994) reported $\Delta \tau \sim 0.04$ on a 1.1 yr baseline. On all of our difference spectra (time baselines of $0.33,0.75$ and $1.08 \mathrm{yr}$ ) our $2-\sigma$ sensitivity is half the level of variations found in Frail at al. (1994), $\Delta \tau \sim 0.02$ at most. We note that no variability was detected at this level in any of later variability experiments.

\subsubsection{PSR B1737+13 (Figure 7)}

With $L_{\perp}=142 \mathrm{AU} \mathrm{yr}^{-1}$ the range of probed spatial scales is from $20 \mathrm{AU}$ to $175 \mathrm{AU}$. No significant variations are found. Frail et al. (1994) reported $\Delta \tau=0.5$ at $4 \mathrm{~km} \mathrm{~s}^{-1}$ (which corresponds to $\Delta\left(I / I_{0}\right) \sim 0.1$ in this relatively deep line) over 0.65 yr. Our $2-\sigma$ sensitivity is $\Delta\left(I / I_{0}\right) \sim 0.2$ for half of baselines, and 0.3 for the rest. Several TSAS features were detected at a similar level in recent experiments, both pulsar and interferometric.

\subsubsection{PSR B1929+10 (Figure 8)}

With $L_{\perp}=37 \mathrm{AU} \mathrm{yr}^{-1}$, the range of probed spatial scales is very narrow, from 6 to $45 \mathrm{AU}$. The absorption profile for this pulsar consists of at least three different velocity components (at $-1.5,5$ and $8 \mathrm{~km} \mathrm{~s}^{-1}$ ). The component at $5 \mathrm{~km} \mathrm{~s}^{-1}$, which is the one with the deepest HI absorption, is the only one exhibiting significant changes. Difference plots show line variations at 3.2 and 3.0- $\sigma$ levels $(\Delta \tau=0.025)$ for time baselines of 0.33 and 1.24 years. We consider these detections to be significant, especially as the same time baselines showed significant variations in EW. This level of variability is the lowest ever detected in TSAS experiments. Both detected difference features are sharp and appear in the same single velocity channel at 5 $\mathrm{km} \mathrm{s}^{-1}$ over different epochs. We further measure and analyze properties of the intervening absorbing clouds traced by these detected features in Section 8 . Baselines of 0.16 , $0.75,0.91$, and 1.08 years have an upper-limit in $\Delta \tau$ of $0.012-0.018$ at this velocity. Frail et al. noticed variations of up to $\Delta \tau \sim 0.03$ for this pulsar, with the largest fluctuations being at a velocity of $\sim 8 \mathrm{~km} \mathrm{~s}^{-1}$, where we observe no significant variations.

As this is the only pulsar in our sample to exhibit significant absorption variations, we have scrutinized the data acquisition and analysis procedure with extra care. For example, a scaling error of approximately $10 \%$ in pulsar strength in a single epoch could result in an erroneous rescaling of optical depths by a similar amount, which would lead to a spurious detection of variation when there is actually none. For example, in Stanimirovic et al (2003), we pointed out an apparent case of this type of problem in Frail et al's spectra of PSR B2016+28. However, it appears that the more advanced CBR spectrometer is indeed able to measure spectra more accurately, as we anticipated. For example, we see no evidence of variations in our experiment with PSR B2016+28 nor with B1133+16, the other particularly bright pulsars in our sample. (Calibration errors would be expected to be most serious in the brighter pulsars, which can easily increase the system temperature by factors of more than two.)

It is notable that B1929+10 is the only pulsar in our sample from which we removed a ghost. Then an obvious question is whether our detected variability could be an artefact of the ghost fitting procedure. We have scrutinized our reduction procedures and remain confident that the variability is real. Spectra for B1929+10 were reduced in the same way as for all other sources. Even the ghost fitting procedure was done in exactly the same way (using exactly the same IDL routines) as the polynomial baseline fitting for other pulsars. The derived error envelopes reflect additional uncertainties due to this fitting process.

In Figure 9 we show all four absorption profiles for this pulsar before (top) and after (bottom) ghost removal. 


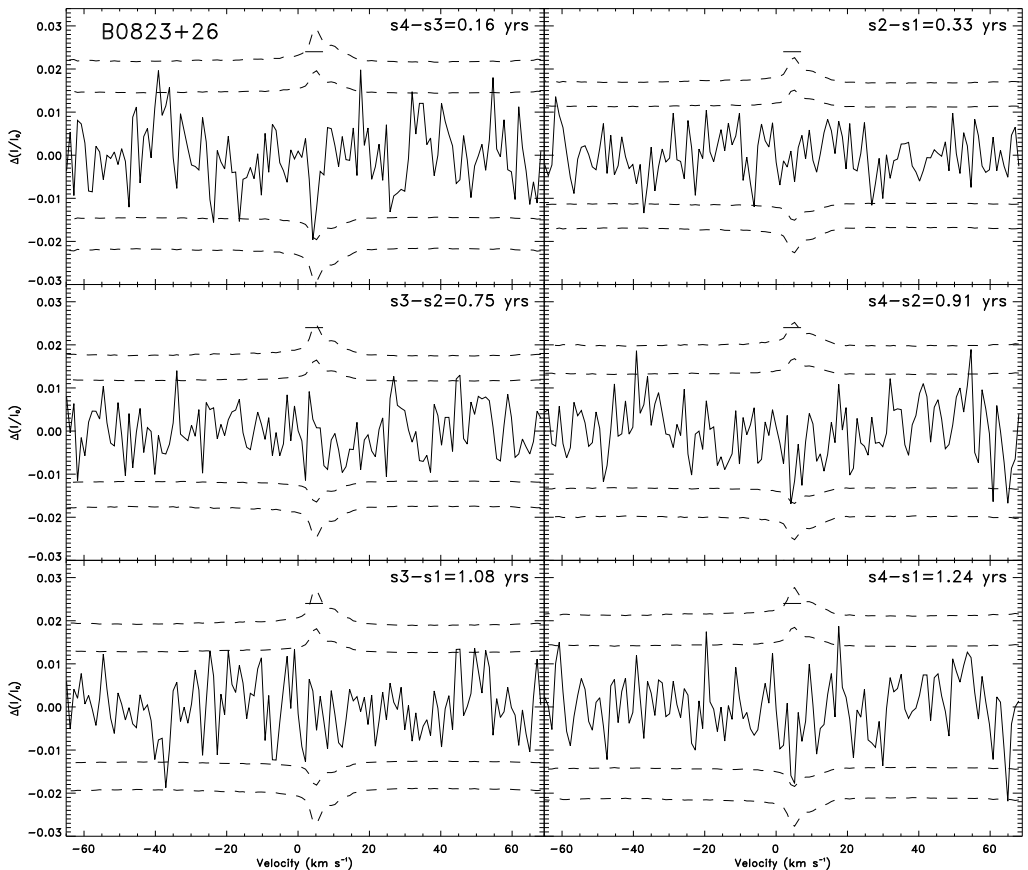

FIG. 5. - Difference spectra for B0823+26 derived from four different observing epochs. Dashed lines show the expected $\pm 2-$ and $3-\sigma$ noise envelopes, whose shape reflects the HI emission in the beam. The vertical scale is in the original units of the absorption spectra, $\Delta\left(I / I_{0}\right)$, which, for small $\tau$, is also equal to $\Delta(\tau)$. The 'on-line' region is shown with a horizontal bar.

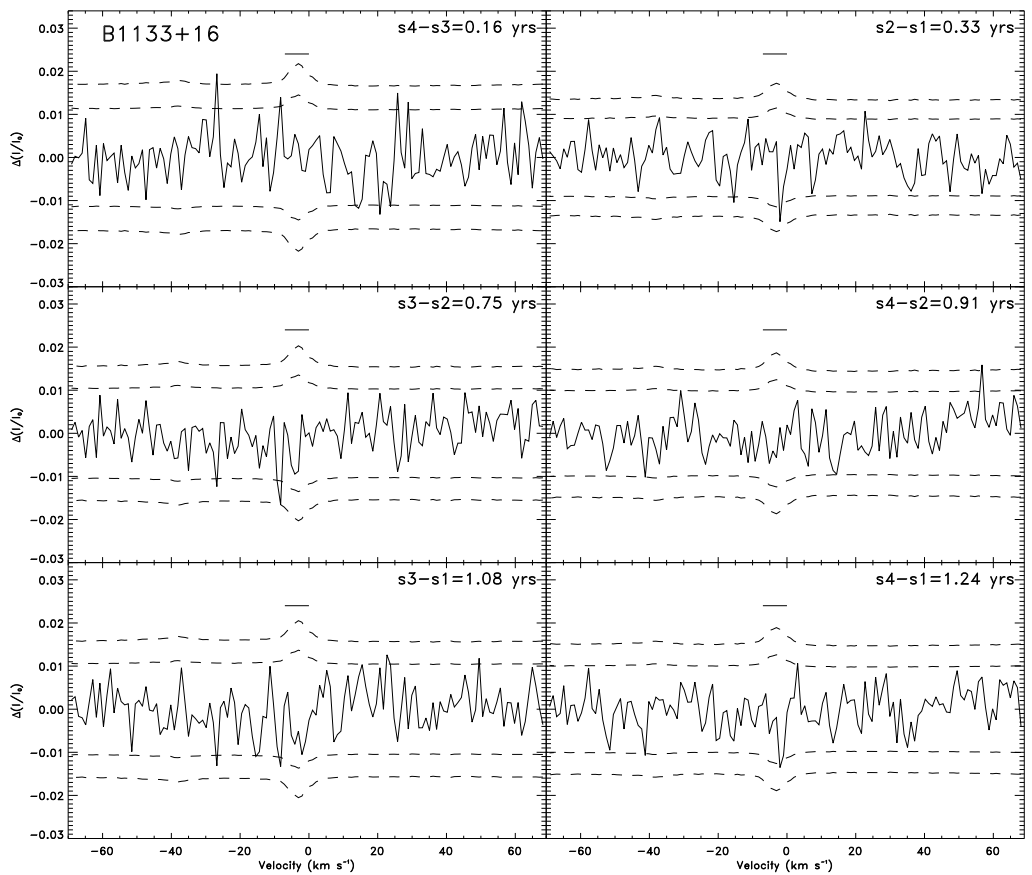

FIG. 6.- Difference spectra for B1133+16 derived from four different observing epochs. See Fig. 5 for further details. 


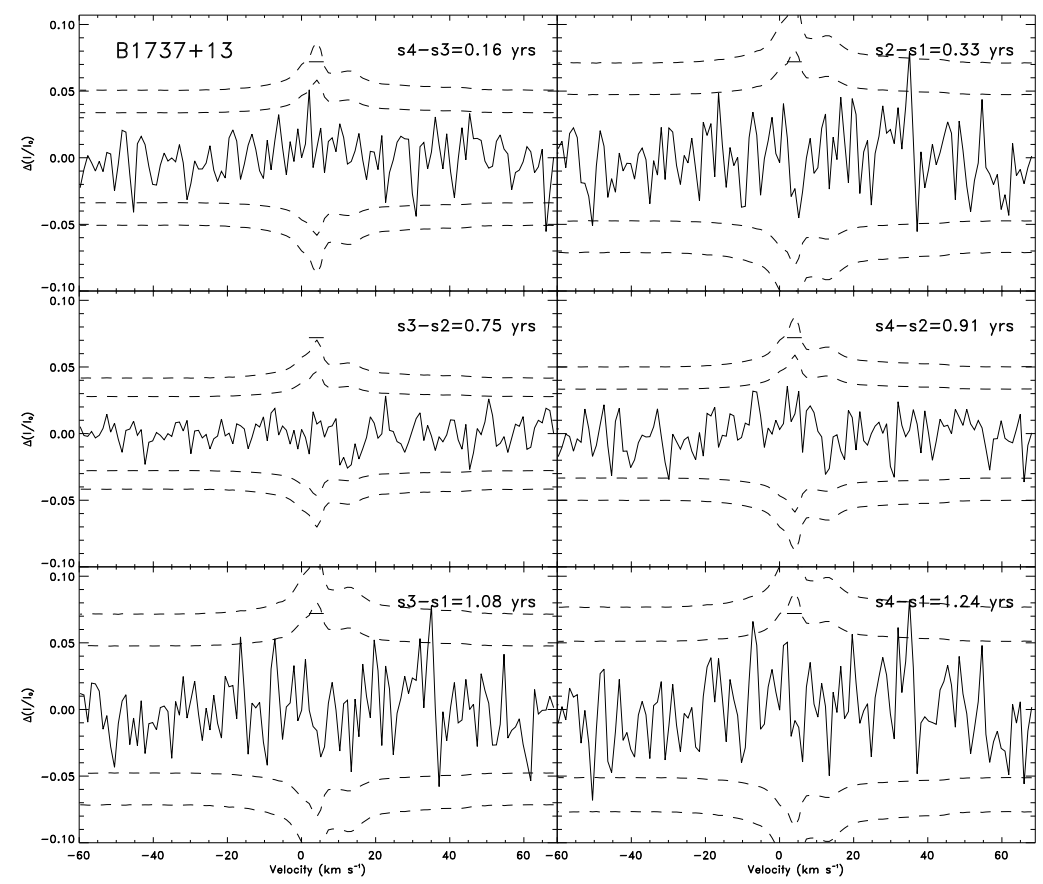

FIG. 7.- Difference spectra for B1737+13 from four different epochs. See Fig. 5 for further details.

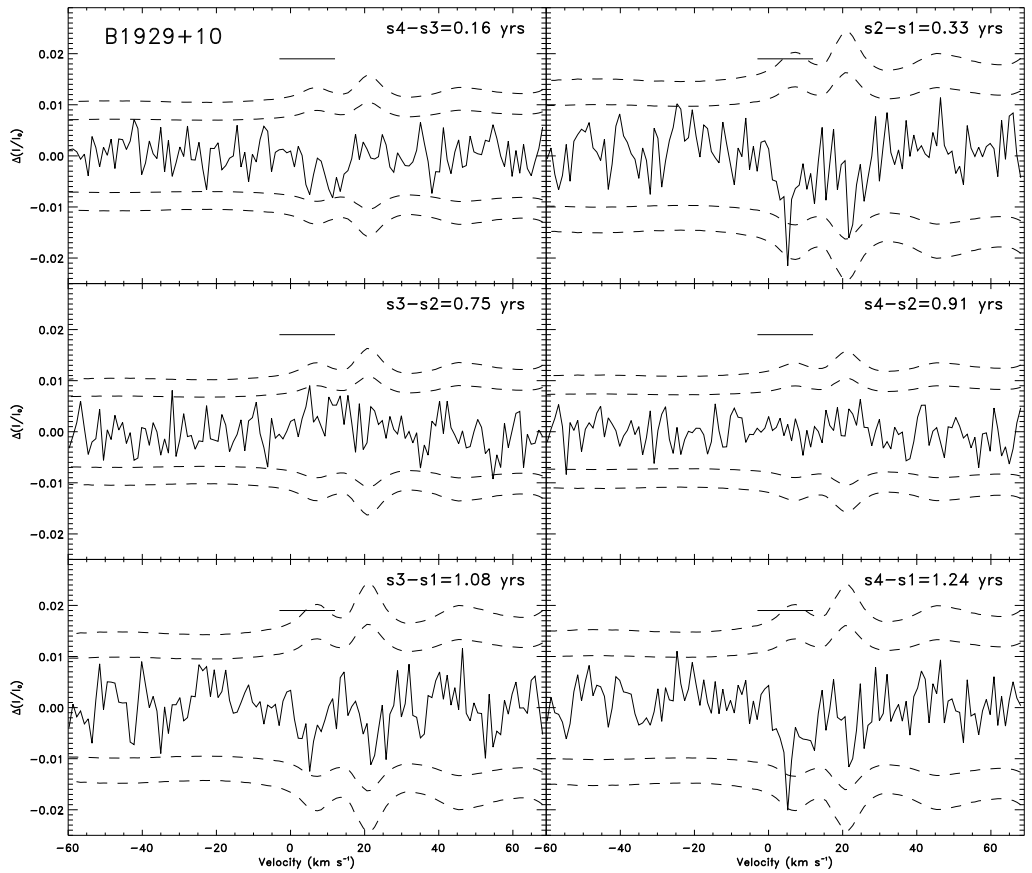

FIG. 8.- Difference spectra for B1929+10 derived from four different observing epochs. Dashed lines show the expected $\pm 2-$ and 3- $\sigma$ noise envelopes, whose shape reflects the HI emission in the beam plus uncertainties associated with ghost excision (See $\$ 3.2$ ). 

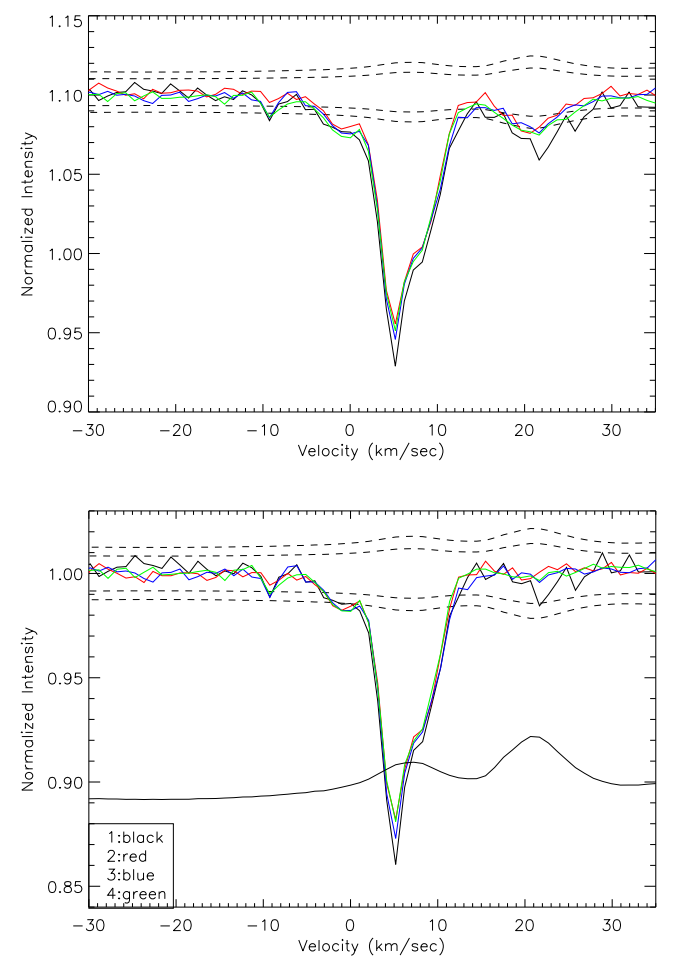

FIG. 9.- HI absorption spectra for B1929+10 from all four epochs before (top) and after (bottom) ghost removal. Dashed lines show the expected \pm 2 - and 3- $\sigma$ noise envelopes for the 1st epoch spectrum The HI emission spectrum, which was exorcized from the displayed absorption spectra (see text), is shown as a faint black line at normalized intensity $\sim 0.92$.

Only the deepest velocity component at $5 \mathrm{~km} \mathrm{~s}^{-1}$ shows variability (both before and after ghost removal), while components at -1.5 and $8 \mathrm{~km} \mathrm{~s}^{-1}$ agree extremely well between epochs. If there were some imperfections related either to a scaling problem or to ghost fitting errors, they would affect all features. We have also examined the estimated coefficients used in equation (2). In the case of the 1 st session spectrum, for example, $A=-4.3 \pm 0.4$. A smaller value for $A$ would result in a larger correction and would bring the 1st session absorption spectrum closer to spectra from other sessions. However, even by taking values within $\pm 2-3 \sigma$ of the best-fit estimate, the observed variations in the difference spectra would still stay significant (above $3-\sigma$ ). The reason for this is that the HI emission at $5 \mathrm{~km} \mathrm{~s}^{-1}$ is not exceptionally high, resulting in a small ghost correction. After all these tests, we remain confident that the detected variability in the case of $\mathrm{B} 1929+10$ is real.

\subsubsection{PSR B2016+28 (Figure 10)}

In Figure 10 we compare all four absorption profiles for this pulsar. This is the slowest-moving pulsar in our sample, so the range of spatial scales covered with our observations is only $1-10$ AUs. No significant variations were found. Our $2-\sigma$ sensitivity is $\Delta\left(I / I_{0}\right) \sim 0.15$ for all baselines. Frail et al. (1994) found $\Delta\left(I / I_{0}\right) \sim 0.15$ (from their Fig. 3) over periods of 0.6 and 1.7 yr. As first noted in Stanimirovic et al. (2003), we compared one of our absorption profiles for this pulsar with data from Frail et al. and concluded that slight calibration problems may have affected Frail et al.'s data. Two recent pulsar experiments found variability at this level but at much larger scales $(>200$ AU; Johnston et al. 2003 and Weisberg et al. 2008).

\subsection{Additional Opacity Fluctuations in Unsmoothed Difference Spectra?}

Weisberg \& Stanimirovic (2007) presented preliminary results of this experiment, and noted that the unsmoothed HI absorption spectra exhibited several apparently significant optical depth variations, mostly in the form of singlechannel fluctuations. The most prominent ones were in the direction of $\mathrm{B} 1929+10$, but there were a few additional variable features toward other pulsars. For the current study, however, where we have applied hanning smoothing to ensure full independence between velocity channels, only the detections in the direction of B1929+10 survive. Could the additional variations in the unsmoothed spectra be real?

The minimum expected temperature for the CNM in thermal equilibrium is 15K (Spitzer 1978; Heiles 2007a). To achieve such a low temperature, dust grain heating of the HI would need to be absent. At this temperature, the full-width, half-maximum (FWHM) of an HI feature will be $0.8 \mathrm{~km} \mathrm{~s}^{-1}$ even in the absence of turbulent broadening; for a less extreme $30 \mathrm{~K}$ the corresponding width is $1.2 \mathrm{~km} \mathrm{~s}^{-1}$. Using a large sample of CNM clouds, Heiles \& Troland (2003b) deduced a relationship between $T_{k}$, the temperature inferred from the measured linewidth, and the spin temperature $T_{s}: T_{k} \sim T_{s}\left(1+M^{2.42}\right)$, where $M$ is the sonic Mach number. For 196 out of $202 \mathrm{CNM}$ components they found that $M>1$, implying then that $T_{k} / T_{s}>1.2$. Therefore, even with the minimum expected 


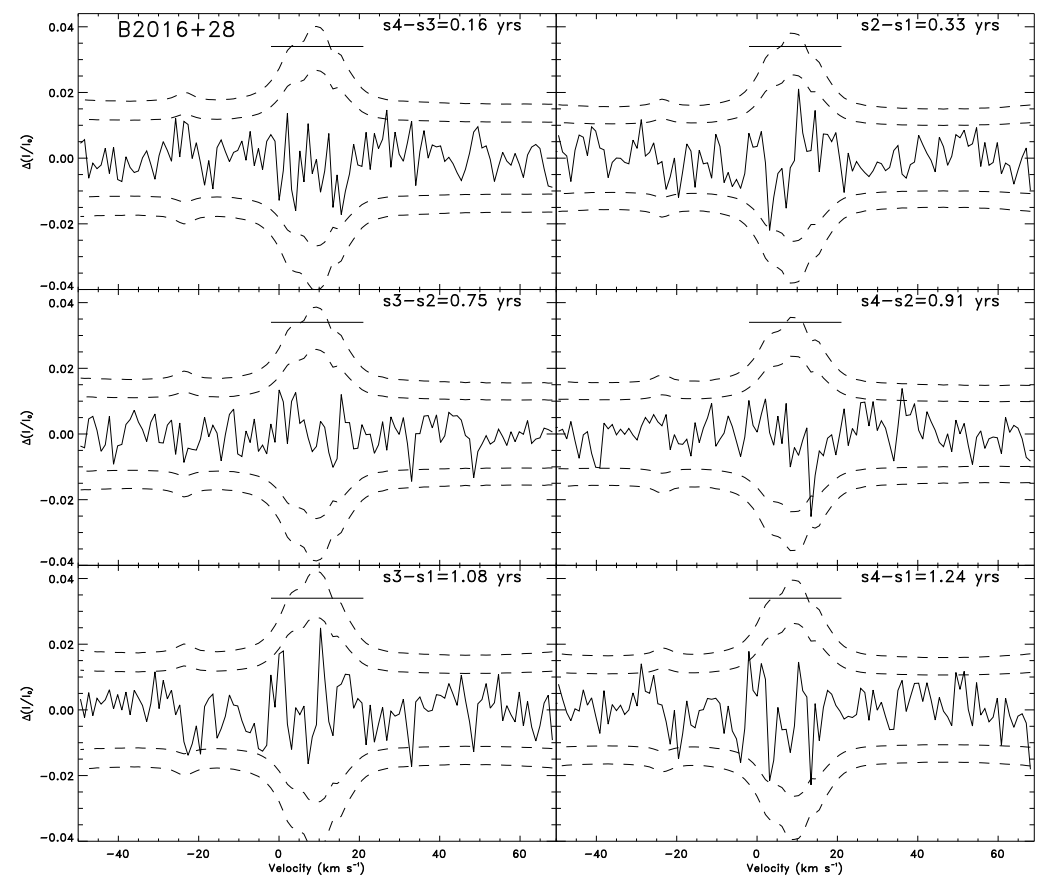

Fig. 10.- Difference spectra for B2016+28 derived from four different observing epochs. See Fig. 5 for further details.

turbulence, the HI absorption line FWHM would be $\sim 0.9$ $\mathrm{km} \mathrm{s}^{-1}$ at $T_{s} \sim 15 \mathrm{~K}$, or $1.3 \mathrm{~km} \mathrm{~s}^{-1}$ at $T_{s} \sim 30 \mathrm{~K}$. So while we have rejected most of Weisberg \& Stanimirović (2007)'s variations because unsmoothed data can be misleading, there are also physical reasons to doubt reality of features with $\mathrm{FWHM}<1 \mathrm{~km} \mathrm{~s}^{-1}$. In contrast, our hanning smoothed resolution appears to match the narrowest plausible TSAS features.

\section{DECOMPOSITION INTO GAUSSIAN COMPONENTS}

In this section we derive the spin temperature and the CNM column density for gas seen in absorption in the direction of our pulsars. We employ the technique developed by Heiles \& Troland 2003a), which is based on fitting Gaussian components to both HI absorption and emission spectra. The purpose of this section is twofold: (i) our further data analysis requires temperatures and column densities of associated CNM clouds; and (ii) since the CNM absorption profiles are often superpositions of several components, our original hope was to search for temporal changes in both the peak optical depth and velocity centroids of all individual velocity components. Unfortunately, since the Gaussian decomposition of absorption and emission profiles is not unique (see discussion in Heiles \& Troland (2003a)), our second goal was not achievable.

To analyze each pulsar, we first selected the epoch with the lowest noise level. The HI absorption and emission spectra were then decomposed into several Gaussian profiles. We note that three of our six pulsars are at high Galactic latitudes and have simple line profiles, easily fitted with one or two Gaussian functions. Optical depth profiles for low latitude pulsars B0540+23, B1929+10, and $\mathrm{B} 2016+28$, (at $b=-3.32,-3.88$, and $-3.98^{\circ}$, respectively), are more complex but still with several obvious velocity components.

\subsection{Procedure for estimating the spin temperature $T_{s}$}

We summarize here very briefly the basic definitions and the procedure, see Heiles \& Troland (2003a) for further details. For each pulsar we have the HI emission profile, $T_{\mathrm{B}}(v)$ (note that this is the emission spectrum that would be observed in the absence of the pulsar) and the HI optical depth spectrum, $I(v) / I_{0}=e^{-\tau(v)}$. Both spectra in general have multiple velocity components.

We first fit $\tau(v)$ with a set of $N$ Gaussian functions using a least-squares technique:

$$
\tau(v)=\sum_{0}^{N-1} \tau_{0, n} e^{-\left[\left(v-v_{0, n}\right) / \delta v_{n}\right]^{2}}
$$

where $\tau_{0, n}$ is the peak optical depth, $v_{0, n}$ is the central velocity, and $\delta v_{n}$ is the $1 / \mathrm{e}$ width of component $n$. $N$ is the minimum number of components necessary to make the residuals of the fit smaller or comparable to the estimated noise level of $I(v) / I_{0}$.

While the optical depth spectrum predominantly reflects the CNM, both the cold and warm neutral media (WNM) contribute to the HI emission spectrum. Consequently we can write the amplitude of the emission spectrum, $T_{\mathrm{B}}(v)$, as follows:

$$
T_{\mathrm{B}}(v)=T_{B, C N M}(v)+T_{B, W N M}(v) .
$$

We now write each of the two terms of Eq. 5 in detail, using the optical depth profile derived from Eq. 4

First, $T_{B, C N M}(v)$, the HI emission originating from $N$ CNM components, can be written as:

$$
T_{B, C N M}(v)=\sum_{0}^{N-1} T_{s, n}\left(1-e^{-\tau_{n}(v)}\right) e^{-\sum_{0}^{M-1} \tau_{m}(v)},
$$

where $T_{s, n}$ is the spin temperature of cloud $n$, and the subscript $m$ represents one of the $M$ CNM clouds that lie in front of cloud $n$. 
Next, $T_{B, W N M}(v)$, the HI emission originating from the WNM, is represented with a set of $K$ Gaussian functions. The complicating factor here is that a certain fraction $F$ of the WNM is located in front of the CNM, while a fraction $(1-F)$ of the WNM is beyond the CNM with its emissions being absorbed by CNM clouds:

$$
T_{B, W N M}(v)=\sum_{0}^{K-1}\left[F_{k}+\left(1-F_{k}\right) e^{-\tau(v)}\right] \times T_{0, k} e^{-\left[\left(v-v_{0, k}\right) / \delta v_{k}\right]^{2}},
$$

where the subscript $k$ corresponds to each of the WNM components and a fraction $F_{k}$ of the WNM cloud $k$ lies in front of all CNM components, while a fraction $1-F_{k}$ is being absorbed by the CNM clouds. For a given order of CNM clouds along the line of sight and a given set of $F_{k}$ values, the $T_{\mathrm{B}}(v)$ profile is simultaneously fitted for Gaussian parameters of the WNM components and the spin temperature of individual CNM clouds.

For each pulsar, we vary the order of Gaussian functions along the line-of-sight (for $N$ CNM components there are $N$ ! possible orderings) and perform the $T_{\mathrm{B}}(v)$ fit. We then choose the ordering of CNM components that gives the smallest residuals in the least-squares fit. Unfortunately, the difference in the fit residuals is often not sufficiently statistically significant to distinguish between different values of $F_{k}$. However, $F_{k}$ has a large effect on the derived spin temperatures. Hence we follow the Heiles \& Troland (2003a) suggestion and estimate the final spin temperatures by assigning characteristic values of $0,0.5$, or 1 to each $F_{k}$ (among the extreme possible values of 0 and 1 ), and repeating this for all possible combinations of WNM clouds. The final spin temperatures are then derived as a weighted average over all trials.

\subsection{Spin temperature and HI column density}

Figure 11 shows results from the Gaussian decomposition and fitting of $\mathrm{HI}$ emission and absorption spectra for two example pulsars, B1737+13 and B1929+10. This figure has three panels: the top panel, displaying the $\mathrm{H}$ I emission spectrum, $T_{B}(v)$, and its decomposition into separate CNM $\left[T_{B, C N M}(v)\right.$, shown as a dotted line $]$ and WNM $\left[T_{B, W N M}(v)\right.$, shown as a dashed line] components; the middle panel giving the optical depth profile and its individual Gaussian-fitted components (shown as dotted lines); and the bottom panel showing the difference between the observed optical depth profile and its Gaussian component fit (dashed lines on this plot show $\pm 1 \sigma$ noise levels). In Table 3 we list the fitted CNM properties.

With the exception of the B1929+10 line of sight, all derived spin temperatures listed in Table 3, are in agreement with what is typically found for CNM clouds (Heiles \& Troland 2003b; Dickey et al. 2003). For example, Heiles \& Troland (2003b) show histograms of WNM and CNM temperatures from their $\mathrm{HI}$ emission/absorption survey of 79 continuum sources. Most CNM clouds have a spin temperature in the range 20-70 K, while CNM clouds with a temperature in the range $150-200 \mathrm{~K}$ appear to be rare. However, spin temperatures we derived for $\mathrm{CNM}$ clouds in the direction of B1929+10 are high, $\sim 150$ and $\sim 200 \mathrm{~K}$. This is the first indication that this particular line of sight could be sampling atypical ISM conditions.

In Table 4 we provide the HI column density in WNM and CNM phases in the direction of each pulsar. The first three pulsars have the $\mathrm{CNM}$ /total column density of about $15-20 \%$, but for $\mathrm{B} 1929+10$ this ratio is only $7 \%$, and for B2016+28 the ratio is about $40 \%$. The very low CNM abundance in the direction of B1929+10 is unusual. For comparison, Heiles \& Troland (2003b) found that all except one of their sources with detected CNM have a CNM fraction $>10 \%$, with a majority of sightlines having $\sim 30 \%$. However, the same study also noted a separate class of objects not exhibiting evidence for the CNM along their lines of sight. They suggested that at least some of these regions may be affected by supershells. The CNM fraction in the direction of B1929+10 is similar to several directions with a CNM fraction of only $2-4 \%$ observed by Stanimirović \& Heiles (2005) and Stanimirović et al. (2007). Clearly, properties of the CNM in the direction of B1929+10 stand out as unusual. As we will see in 97 . a significant fraction of this line of sight is located inside the Local Bubble.

\section{A STUDY OF THE LINES OF SIGHT}

We have examined the lines of sight of all pulsars in our sample. While $\mathrm{B} 1737+13$ and $\mathrm{B} 2016+28$ are at a significant distance from the Sun and their lines of sight probably average over many interstellar clouds, B0823+26, $\mathrm{B} 1133+16$ and $\mathrm{B} 1929+10$ are close with significant portions of their lines of sight probing the very local ISM. We detail here some interesting properties of the latter three pulsars' lines of sight.

PSR B1929+10 is the closest pulsar in our sample, $D=361_{-10}^{+8}$ pc (Chatteriee et al. 2004), and this relatively close distance raises the question of whether the observed fluctuations of HI optical depth are somehow related to the pulsar's proximity to the solar system. For example, we are embedded near the middle of a low-density, hightemperature region called the Local Cavity which extends 1-200 pc before being bounded by a high density wall in most directions, so a significant portion of the line of sight lies inside it. In what follows, the "Local Bubble (LB)" consists of the Local Cavity plus the wall. Lallement et al. (2003) mapped the dense neutral gas wall bounding the Local Cavity via interstellar absorption measurements of stars of known distance. The updated map of the neutral gas surrounding the Local Cavity by Welsh et al. (2010a) confirms that the line of sight from B1929+10 enters the wall of the LB at a distance of $\sim 120 \mathrm{pc}$ and then pierces the wall for $\sim 50-60$ pc. A lower-resolution interstellar reddening map from Toscano et al. (1999) also indicates that the LB boundary is distended significantly toward the pulsar. Furthermore, the B1920+10 line of sight appears to be in or near a long extended neutral finger of the LB (possibly an interstellar tunnel gouged by a supernova) and may therefore lie in the finger or along its wall for some distance. The dense finger is most likely associated with the Ophiuchus molecular cloud and de Geus et al. (1990) found that the $\mathrm{CO}$ distribution in this region is filamentary and has radial velocities in the range $4.2-5.3 \mathrm{~km} \mathrm{~s}^{-1}$ (note that our varying $\mathrm{HI}$ component is centered at $4.8 \pm 0.1$ $\mathrm{km} \mathrm{s}^{-1}$ ).

In addition to the above, we found that two stars within 3 degrees from B1929+10 have been observed in NaI: HD178125 is at a distance of $173 \mathrm{pc}$, while HD180555 is at a distance of only $106 \mathrm{pc}$. Based on the data presented in 
TABLE 3

CNM COMPONENTS FROM GAUSSIAN DECOMPOSITION.

\begin{tabular}{lccccc}
\hline \hline PSR & $\tau_{0}$ & $\begin{array}{c}v_{0}(\mathrm{LSR}) \\
\left(\mathrm{km} \mathrm{s}^{-1}\right)\end{array}$ & $\begin{array}{c}\Delta v \\
\left(\mathrm{~km} \mathrm{~s}^{-1}\right)\end{array}$ & $\begin{array}{c}T_{\text {spin }} \\
(\mathrm{K})\end{array}$ & $\begin{array}{c}\mathrm{N}(\mathrm{HI})_{\mathrm{CNM}} \\
\left(10^{20} \mathrm{~cm}^{-2}\right)\end{array}$ \\
\hline B0823+26 & & & & & \\
& $0.26 \pm 0.01$ & $4.91 \pm 0.02$ & $2.3 \pm 0.1$ & $62 \pm 5$ & $0.72 \pm 0.02$ \\
B1133+16 & & & & & \\
& $0.16 \pm 0.02$ & $-2.10 \pm 0.03$ & $1.7 \pm 0.1$ & $27 \pm 5$ & $0.15 \pm 0.01$ \\
& $0.16 \pm 0.01$ & $-3.5 \pm 0.1$ & $3.6 \pm 0.1$ & $48 \pm 6$ & $0.53 \pm 0.03$ \\
B1737+13 & & & & & \\
& $0.05 \pm 0.01$ & $0.7 \pm 0.6$ & $4.2 \pm 0.8$ & $59 \pm 6$ & $0.25 \pm 0.03$ \\
& $0.78 \pm 0.13$ & $4.19 \pm 0.06$ & $2.1 \pm 0.1$ & $35 \pm 7$ & $1.1 \pm 0.1$ \\
& $0.66 \pm 0.13$ & $4.59 \pm 0.05$ & $1.2 \pm 0.1$ & $22 \pm 6$ & $0.33 \pm 0.05$ \\
B1929+10 & & & & & \\
& $0.017 \pm 0.002$ & $-0.7 \pm 0.3$ & $5.3 \pm 0.8$ & $199 \pm 25$ & $0.35 \pm 0.04$ \\
& $0.120 \pm 0.005$ & $4.8 \pm 0.1$ & $3.0 \pm 0.1$ & $148 \pm 4$ & $1.00 \pm 0.04$ \\
& $0.076 \pm 0.003$ & $8.4 \pm 0.2$ & $3.9 \pm 0.3$ & $206 \pm 7$ & $1.15 \pm 0.06$ \\
B2016+28 & & & & & \\
& $1.12 \pm 0.03$ & $3.70 \pm 0.03$ & $2.36 \pm 0.06$ & $50 \pm 30$ & $2.8 \pm 0.3$ \\
& $0.67 \pm 0.04$ & $5.97 \pm 0.04$ & $1.4 \pm 0.1$ & $50 \pm 30$ & $0.9 \pm 0.1$ \\
& $0.81 \pm 0.05$ & $9.16 \pm 0.06$ & $10.2 \pm 0.2$ & $80 \pm 50$ & $13.3 \pm 1.5$ \\
& $0.57 \pm 0.05$ & $9.70 \pm 0.04$ & $2.7 \pm 0.2$ & $50 \pm 30$ & $1.7 \pm 0.2$ \\
& $1.30 \pm 0.03$ & $13.3 \pm 0.01$ & $1.54 \pm 0.03$ & $20 \pm 10$ & $0.7 \pm 0.1$ \\
\hline
\end{tabular}

TABLE 4

Hi Column Densities in CNM ANd WNM toward Pulsars

\begin{tabular}{ccccc}
\hline \hline PSR & $\begin{array}{c}N(\mathrm{HI})_{\mathrm{WNM}} \\
\left(10^{20} \mathrm{~cm}^{-2}\right)\end{array}$ & $\begin{array}{c}N(\mathrm{HI})_{\mathrm{CNM}} \\
\left(10^{20} \mathrm{~cm}^{-2}\right)\end{array}$ & $\begin{array}{c}N(\mathrm{HI})_{\mathrm{TOT}} \\
\left(10^{20} \mathrm{~cm}^{-2}\right)\end{array}$ & $N(\mathrm{HI})_{\mathrm{CNM}} / N(\mathrm{HI})_{\mathrm{TOT}}$ \\
\hline B0823+26 & 4.3 & 0.7 & 5.0 & 0.14 \\
$\mathrm{~B} 1133+16$ & 3.5 & 0.7 & 4.2 & 0.16 \\
$\mathrm{~B} 1737+13$ & 6.3 & 1.7 & 8.0 & 0.21 \\
$\mathrm{~B} 1929+10$ & 34.7 & 2.5 & 37.2 & 0.07 \\
$\mathrm{~B} 2016+28$ & 28.9 & 18.7 & 47.7 & 0.39 \\
\hline
\end{tabular}


Genova et al. (1997), these stars show NaI absorption at an LSR velocity of +5.9 and $+5.6 \mathrm{~km} \mathrm{~s}^{-1}$, respectively (velocity uncertainty for these measurements is $1-1.5 \mathrm{~km} \mathrm{~s}^{-1}$ ). As our varying pulsar absorption component is centered at $+4.8 \mathrm{~km} \mathrm{~s}^{-1}$ (with a FWHM of $3 \mathrm{~km} \mathrm{~s}^{-1}$ ), it is most likely tracing the same interstellar cloud seen in the direction of the two HD stars. This would imply that the absorbing cloud is at a distance of $<106 \mathrm{pc}$, as it is seen against HD180555, and therefore embedded in the Local Cavity or the LB wall.

The Local Cavity itself is a region of low scattering; while most investigations have concluded that there is enhanced scattering at its boundary, implying enhanced turbulence there (Phillips \& Clegg 1992; Bhat et al. 1998; Gupta et al. 1999; Chatteriee et al. 2001). Based on modeling of many NaI absorption features, Genova et al. (1997) found that the +5.9 and $+5.6 \mathrm{~km} \mathrm{~s}^{-1}$ absorption components in the direction of HD178125 and HD180555 belong to a plane-parallel flow immersed in the Local Cavity, the so-called "flow C". They suggested that flow $\mathrm{C}$ could represent gas condensing out of the LB wall. Generally, many diffuse interstellar clouds (so-called "Local Fluff") have been found inside the Local Cavity itself and have been interpreted as signatures of the development of Rayleigh-Taylor instabilities in the wall, whereby gas is condensing out of the leading edge of the LB wall and flowing toward the Sun (Müller et al. 2006; Breitschwerdt \& de Avillez 2006).

To examine lines of sight toward B0823+26 and B1133+16, we turn to Figure 7 of Lallement et al. (2003) which shows neutral gas in vertical planes at fixed galactic longitudes. In the case of $\mathrm{B} 0823+26\left(b \sim 32^{\circ}\right)$, about one-half of the line of sight appears to be inside the Local Cavity and traverses the LB wall over only a short distance. In the case of $\mathrm{B} 1133+16\left(b \sim 69^{\circ}\right)$, the line of sight appears to exit the LB through an opening or a chimney of low density material rather than a bounding wall or any other substantially dense neutral material.

In summary, since almost one-half of the line of sight toward B1929+10 is associated with the LB and the absorbing gas is likely to be within $106 \mathrm{pc}$, we hypothesize that our detected TSAS clouds along this line of sight are formed or influenced by the LB. Based on the stellar NaI absorption lines at similar velocities to those we find in HI toward B1929+10, we conclude that our TSAS fluctuations are likely to be associated with the LB wall. The high spin temperature of the absorbing clouds, as shown in the previous section, may result from the thermally unstable gas due to wall fragmentation (see also Section 9.4). It is then not surprising that we did not detect TSAS in the direction of B0823+26 and B1133+16, despite equally good sensitivity, because these lines of sight do not traverse much or any of the wall.

\section{TSAS PROPERTIES INFERRED FROM OBSERVATIONS}

In Sections 4 and 5 we have identified several significant variations in absorption profiles that could be interpreted as TSAS. All of these features are in the direction of B1929+10. We summarize their observed properties in Table 5. We measure TSAS properties from EW variations, as this is more robust than using difference spectra which have a lower $\mathrm{S} / \mathrm{N}$. We estimate TSAS properties from EW variations with $\gtrsim 2.5-\sigma$.

Table 5 lists the following quantities: the time baseline over which the change in EW is observed, the corresponding transverse spatial scale, the absolute value of $\Delta \mathrm{EW}$, the significance of the fluctuation, the HI column density of the TSAS $\left(N(\mathrm{HI})_{\text {TSAS }}\right)$, the inferred HI volume density of TSAS (assuming spherical HI blobs whose radius is equal $l$ ), and the inferred thermal pressure. Some comments are necessary for interpreting these tables. (i) Our observations do not constrain the exact size of TSAS, but rather its characteristic scale. In measuring the tranverse distance $(l)$ traveled by the pulsar over a time span exhibiting a variation in $\mathrm{HI}$ absorption spectra, we are sampling a spatial scale over which we have sampled the optical depth function. Therefore, even in the case that significant fluctuations are due to discrete blobs of gas, $l$ does not necessarily correspond to a physical size of these blobs. This point has been emphasized by Deshpande (2000).

(ii) The temperature of TSAS, needed to estimate $N(\mathrm{HI})_{\text {TSAS }}$, is also not constrained directly by observations. We have estimated in Section 6 the temperature of the parent CNM clouds with which TSAS is associated. In the scenario where TSAS results from discrete, elongated objects, such as in Heiles (1997) and Heiles (2007b), CNM clouds are constituted from TSAS and an interTSAS medium. The derived spin temperature in this case corresponds to the inter-TSAS medium, while TSAS could have a much colder temperature of $\sim 15-30 \mathrm{~K}$ (Heiles 1997).

There are several interesting results:

1. Significant fluctuations (Table 5) are found on a variety of spatial scales $l=\frac{D_{\mathrm{HI}}}{350 \mathrm{pc}} \times(6,12,28,46)$ AU (with $D_{\mathrm{HI}}$ being a TSAS distance), suggesting that the CNM in the direction of B1929+10 has structure on spatial scales of 5-50 AUs. Our characteristic scales for TSAS are similar to results from VLBA experiments: Brogan et al. (2005) found a typical size of $25 \mathrm{AU}$ and Lazio et al (2009) found a typical size of $10 \mathrm{AU}$.

2. If we assume that TSAS has the same spin temperature as its parent CNM cloud $(\sim 170 \mathrm{~K}$ in the case of B1929+10), we estimate a HI column density of $N(\mathrm{HI})_{\mathrm{TSAS}}=(1-3) \times 10^{19} \mathrm{~cm}^{-2}$. This is slightly lower than the typical CNM column density of $5 \times 10^{19} \mathrm{~cm}^{-2}$ found for the CNM in the Heiles $\&$ Troland (2003b) survey.

3. All TSAS features contribute 10-30 percent to the HI column density of their 'parent' CNM clouds.

4. If we interpret TSAS as being in the form of spherically symmetric blobs, its observationally inferred HI volume density and thermal pressure are $>10^{4}$ $\mathrm{cm}^{-3}$ and $>$ a few $\times 10^{6} \mathrm{~cm}^{-3} \mathrm{~K}$, respectively. These values are more than two orders of magnitude larger than what is typically expected for the ISM, in agreement with previous TSAS measurements (see Section 2).

5. In terms of HI mass, TSAS contains only $\sim 10^{-3}$ Earth masses. This is many orders of magnitude 

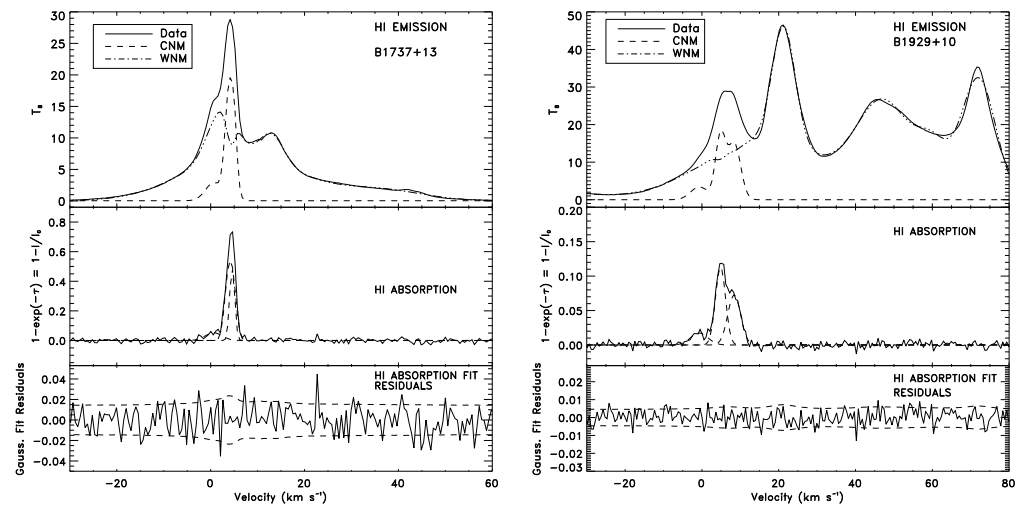

FIG. 11. - Left: Fitted results for the opacity profile and H I emission for B1737+13 observed at epoch 2001.86. Right: Fitted results for the opacity profile and H I emission for B1929+10 observed at epoch 2000.95. (Top) The H I emission profile is shown with the solid line. The total H I emission from WNM components is shown with the dot-dot-dashed line. The dashed line shows the emission contribution from all CNM components $\left(T_{B, C N M}(v)\right)$. (Middle) The optical depth profile is shown with the solid line. Fitted Gaussian components are shown with dashed lines. (Bottom) Residuals after fitting Gaussian function(s) to the optical depth profile. The overlaid contours show $\pm 1-\sigma$ levels.

below what is required for gravitationally confined clouds.

6. In the shaped TSAS model (Heiles et al. 1997) TSAS could be much colder with a temperature up to a factor of 10 lower than what we have used. This would reduce the derived $N(\mathrm{HI})_{\mathrm{TSAS}}$ and $n(\mathrm{HI})$ by a factor of 10 , and $P / k$ by a factor of 100 . If an elongation factor $G \sim 5-10$ is further introduced, the derived thermal pressure can be brought down to $P / k \sim 2 \times 10^{4} \mathrm{~cm}^{-3} \mathrm{~K}$ which is close to the traditional hydrostatic pressure (with some turbulent contribution). However, this could work only for the case of both low TSAS temperature and significant line-of-sight elongation.

In Table 6] we provide upper limits on TSAS properties based on non-detections in the directions of four other pulsars.

\section{DISCUSSION}

\subsection{The Heiles (1997) discrete cloud model}

In the Heiles (1997) scenario, a typical CNM cloud consists of an inter-TSAS medium and many discrete TSAS features in the form of cylinders or disks, which are homogeneously and isotropically distributed within the cloud. An arbitrary line of sight samples many cylinders or disks; however only cylinders seen end-on or disks seen edge-on have sufficient column densities to produce absorption features recognized as TSAS. Meanwhile, non-end-on cylinders or non-edge-on disks have very little column density and blend to form a smooth background which contributes very little to the measured $\mathrm{HI}$ absorption profile. The model assumed the following basic parameters for TSAS: a cloud size perpendicular to the line of sight $L_{\perp}=30$ $\mathrm{AU}, T_{\mathrm{s}}=15 \mathrm{~K}, N(\mathrm{HI})_{\mathrm{TSAS}}=0.33 \times 10^{19} \mathrm{~cm}^{-2}$, and $\aleph=3$ for the number of end-on cylinders or edge-on disks along every line of sight. Two values were considered for $r$, the ratio of the CNM to TSAS column density: $r=N(\mathrm{HI})_{\mathrm{CNM}} / N(\mathrm{HI})_{\mathrm{TSAS}}=30$ and 100 . The model predicts the elongation factor $G$ and the volume filling factor $\phi$ of TSAS required to ameliorate the over-pressure prob- lem, under the assumption that the asymmetric TSAS features are uniformly and isotropically distributed throughout CNM clouds. If TSAS is in the form of edge-on disks then $G \sim 10$ and they fill about $3.3 \%$ of the CNM volume; whereas if TSAS is in the form of end-on cylinders then $G \sim 3.8$ and they fill about $3.6 \%$ of the CNM volume. However, the volume filling factor of TSAS is directly proportional to the number of end-on cylinders or edge-on disks $\aleph$ along any line of sight.

If we assume $T_{s}=15 \mathrm{~K}$ for our TSAS detections, a geometric factor $G=5-10$ is required to explain the observed TSAS volume density and pressure within the context of the Heiles model. This is in the range proposed by Heiles (1997). In addition, the TSAS HI column density as well as the $r$ ratio are similar to values explored in the model. It is therefore reasonable to compare our estimated volume filling factor of TSAS with the model predicted values.

In the case of B1929+10, as the pulsar moves through the ISM our HI absorption spectra have essentially sampled a one-dimensional cut across the absorbing CNM cloud (about $50 \mathrm{AU}$ long) and detected at least two TSAS features. This means that a one-dimensional covering fraction of TSAS in this direction is at least $40 \%$, if we assume a 10AU plane-of-sky TSAS size. We can now estimate the fraction of the line of sight occupied by TSAS. We first estimate the line-of-sight size of the parent CNM cloud. As this cloud has $N(H I) \sim 10^{20} \mathrm{~cm}^{-2}$ (from Table 3), and if we assume a CNM volume density of $100 \mathrm{~cm}^{-3}$, this results in a length of about $7 \times 10^{4}$ AU. Assuming a cylindrical TSAS with a $10 \mathrm{AU}$ plane-of-sky size and a geometrical elongation $G=10$, results in the volume filling factor of $<0.2 \%$ in the direction of B1929+10. The volume filling factor in the case of other pulsars in our experiment is zero. We note that our sensitivity for B $0823+26$ and B1133+16 is equally good as for B1929+10, and all three pulsars probe interstellar environments with a similar optical depth (peak of $\sim 0.2$, see Figures 1 and 2) and a similar line-of-sight length. In a similarly sensitive experiment, Minter et al. (2005) had 18 observing epochs for one pulsar, about 150 trials, and no detections.

The observationally inferred volume filling factor of TSAS in the direction of our pulsars $(0$ or $<0.2 \%)$ is lower 
than the model prediction $(\sim 3 \%)$. More importantly, we observe significant inhomogeneity in the galactic distribution of TSAS: no detections toward four pulsars, and a high detection rate toward B1929+10. For the Heiles (1997) model to remain viable, our observations require inclusion of the possibility of different TSAS properties (specifically $L_{\perp}, G$ and $\aleph$ ) along different lines of sight, and/or additional local physical processes which can modify the TSAS production rate. For example, a highly elongated TSAS with $L_{\perp}$ a million times smaller than the size of its parent CNM cloud would theoretically result in a volume filling factor close to zero. While in principle this scenario could work, we are still left with the puzzle of what physical processes can produce such tiny clouds and at the same time greatly vary cloud properties across the ISM.

\subsection{The shocked CNM model (Hennebelle \& Audit 200\%)}

Several recent numerical simulations of the small-scale structure in the ISM have addressed the TSAS phenomenon. In particular, Hennebelle \& Audit (2007) performed relatively high resolution numerical simulations of colliding WNM flows and the formation of CNM clouds. A collision of incoming WNM streams creates a thermally unstable region of higher density and pressure but lower temperature, which further fragments into cold structures. The thermally unstable gas has filamentary morphology and its fragmentation into cold clouds is promoted and controlled by turbulence. While typical CNM clouds are formed in these simulations within $\sim 1 \mathrm{Myr}$, transient shocked CNM regions, produced by supersonic collisions between CNM fragments, are also found, with properties similar to those of TSAS: $n \sim 10^{3-4} \mathrm{~cm}^{-3}, T \sim 30 \mathrm{~K}$, $P / k \sim 10^{5} \mathrm{~K} \mathrm{~cm}^{-3}$, and a slightly larger size of $\sim 400$ AU. Hennebelle et al. (2007) found that $10 \%$ of lines of sight cross dense CNM gas with $n>10^{3} \mathrm{~cm}^{-3}$, and only $0.1 \%$ of lines of sight cross gas denser than $n>10^{4} \mathrm{~cm}^{-3}$, suggesting that extremely dense TSAS should rarely be detected.

While this was a 2D simulation of a coherent region only $20 \times 20$ pc large, basic observed properties of the CNM/WNM were surprisingly well captured. For example, the CNM column density peak, and the HI column density distribution function appear close to observed properties based on the Heiles \& Troland (2003) survey. Considering that the simulation has a resolution of 400 AU (cruder than the scales generally sampled in pulsar and interferometer experiments) and probes regions only 20 pc across (much smaller than our lines of sight), direct comparison with our observations is not straightforward. Nevertheless, the predicted properties of the shocked CNM clouds, especially cloud abundance, are encouraging. Considering that the CNM/TSAS production in converging flows is controlled by the level of turbulence in colliding flows, local turbulent enhancements may be able to provide the necessary tuning and therefore explain the inhomogeneity in the spatial distribution of TSAS observed in the direction of $\mathrm{B} 1929+10$.

\subsection{The cut-off scale of the CNM turbulence?}

In an attempt to compare various observations, we have investigated HI TSAS detections and upper limits from all published and recent pulsar and interferometric experiments as a function of their spatial scale. The results are summarized in Figures 12 and 13.

In Figure 12 we first show the maximum variation in HI optical depth $(\Delta \tau)$ as a function of peak optical depth along the line of sight $\left(\tau_{\text {peak }}\right)$. This plot separates all sources cleanly into two groups: "low optical depth" with $\tau_{\text {peak }}<0.5(\mathrm{~B} 1929+10, \mathrm{~B} 0823+16, \mathrm{~B} 1133+16$, and B0301+19), and "high optical depth" with $\tau_{\text {peak }}>0.5(3$ interferometric detections, pulsars B1737+13 B2016+28 from this study, highly sampled pulsar B0329+54 from Minter et al. 2005, and B0736-40, B1451-68, B1557-50 from Johnston et al. 2003). The only exception in this figure is the 2.6- $\sigma$ detection by Weisberg et al. (2008) in the case of B0301+19 which probes a low optical depth $\mathrm{CNM}$ but has $\Delta \tau \sim 0.1$. This plot suggests that regions with high optical depth (and therefore high CNM column density) have higher level of $\Delta \tau$ variations than regions with low CNM column density. In other words, this implies that the the level of $\tau$ fluctuations tracing TSAS is intimately associated with the larger scale structure of the parental CNM.

Figure 13 shows the detected level of optical depth variations, or upper limits, as a function of TSAS spatial scale $l$. No obvious correlation between the two quantities is noticeable. The Kendall's tau correlation test for censored data (Isobe et al. 1986) gives a probability of $62 \%$ that the correlation is not present. The low and high $\tau_{\text {peak }}$ groups of sources nicely separate in this plot around $\Delta \tau=0.03$ : the high- $\tau_{\text {peak }}$ sources are above this line, while all low$\tau_{\text {peak }}$ sources except B0301 are below this line. We investigated whether a correlation between TSAS scale $l$ and $\Delta \tau$ exists for the two groups separately. For both groups the Kendall's tau correlation test for censored data gives a probability of $\sim 50 \%$ that the correlation is not present.

We therefore conclude that the detected level of optical depth variations, as well as upper limits, do not show evidence for a correlation between $l$ and $\Delta \tau$. Such a correlation, with $\Delta \tau \propto l^{(\alpha-2) / 2}$, is expected in the Deshpande (2000) power law model, and would represent the tail-end of the turbulent spectrum of the HI optical depth on larger scales (0.02 to $4 \mathrm{pc}$, Deshpande et al. 2000). We overplot this predicted level of variations in Figure 13 as a sloping line.

Our compilation of recent interferometric and pulsar observations of TSAS does not provide evidence for the power-law turbulent spectrum of $\Delta \tau$ on scales $<1$ to 1000 AU. While various sources probe separate lines of sight, we note that $\mathrm{B} 0823+26, \mathrm{~B} 1133+16$ and $\mathrm{B} 1929+10$ all probe the local ISM (within $400 \mathrm{pc}$ ), with similar peak optical depth, and with very similar sensitivity. This essentially provides an almost uniformly sampled region on this graph from $\sim 10$ to $200 \mathrm{AU}$. Similar arguments apply to the higher optical depth portion of this diagram. We are left with the conclusion that TSAS is a sporadic phenomenon.

It is interesting to note that the smallest spatial scale of TSAS detections in both interferometric and B1929+10 studies is $\sim 10$ AU. Two pulsars $(B 2016+28$ and B0329+54) have probed scales $<10$ AU, however TSAS was not detected. The spatial scales of $\sim 10 \mathrm{AU}$ for TSAS in the CNM are especially interesting, since in the case of hydrodynamic turbulence the cut-off scale of 
TABLE 5

ObSERVED AND DERIVED PROPERTIES OF TSAS FEATURES WITH $\gtrsim 2.5-\sigma$ IN THE DIRECTION OF B1929+10.

\begin{tabular}{ccccccc}
\hline \hline $\begin{array}{c}\Delta t \\
(\mathrm{yr})\end{array}$ & $\begin{array}{c}\mathrm{AU}) \\
(\mathrm{AUmW} \mid\end{array}$ & $\begin{array}{c}\mid \Delta \mathrm{Em} \mathrm{s}) \\
\left(\mathrm{km}^{-1}\right)\end{array}$ & $\begin{array}{c}\text { Signif. } \\
(\sigma)\end{array}$ & $\begin{array}{c}N(\mathrm{HI})_{\mathrm{TSAS}}^{a} \\
\left(10^{19} \mathrm{~cm}^{-2}\right)\end{array}$ & $\begin{array}{c}n(\mathrm{HI})^{b} \\
\left(10^{4} \mathrm{~cm}^{-3}\right)\end{array}$ & $\begin{array}{c}P / k \\
\left(10^{6} \mathrm{~cm}^{-3} \mathrm{~K}\right)\end{array}$ \\
\hline 0.16 & 6 & 0.046 & 2.8 & 1.4 & 16 & 27 \\
0.33 & 12 & 0.083 & 3.3 & 2.6 & 14 & 24 \\
0.75 & 28 & 0.040 & 2.5 & 1.2 & 3 & 5 \\
1.24 & 46 & 0.089 & 3.5 & 2.8 & 4 & 7 \\
\hline
\end{tabular}

${ }^{a}$ We have assumed $T_{s}=170 \mathrm{~K}$ based on Table 3

${ }^{b}$ Calculated assuming a spherical geometry.

TABLE 6

UPPER LIMITS ON OBSERVED TSAS PROPERTIES FROM NON-DETECTIONS.

\begin{tabular}{cccccc}
\hline \hline PSR & $\begin{array}{c}l \\
\mathrm{AU})\end{array}$ & $\begin{array}{c}|\Delta \mathrm{EW}| \\
\left(\mathrm{km} \mathrm{s}^{-1}\right)\end{array}$ & $\begin{array}{c}N(\mathrm{HI})_{\mathrm{TSAS}}^{a} \\
\left(10^{18} \mathrm{~cm}^{-2}\right)\end{array}$ & $\begin{array}{c}n(\mathrm{HI})^{b} \\
\left(10^{4} \mathrm{~cm}^{-3}\right)\end{array}$ & $\begin{array}{c}P / k \\
\left(10^{6} \mathrm{~cm}^{-3} \mathrm{~K}\right)\end{array}$ \\
\hline B0823+26 & $5-50$ & 0.05 & $<6$ & $<7$ & $<4$ \\
B1133+16 & $20-170$ & 0.06 & $<4$ & $<2$ & $<1$ \\
B1737+13 & $20-180$ & 0.35 & $<19$ & $<7$ & $<4$ \\
B2016+28 & $1-10$ & 0.45 & $<41$ & $<270$ & $<140$ \\
\hline
\end{tabular}

${ }^{a}$ We have assumed $T_{s}=50 \mathrm{~K}$.

${ }^{b}$ Calculated assuming a spherical geometry and the largest probed size.

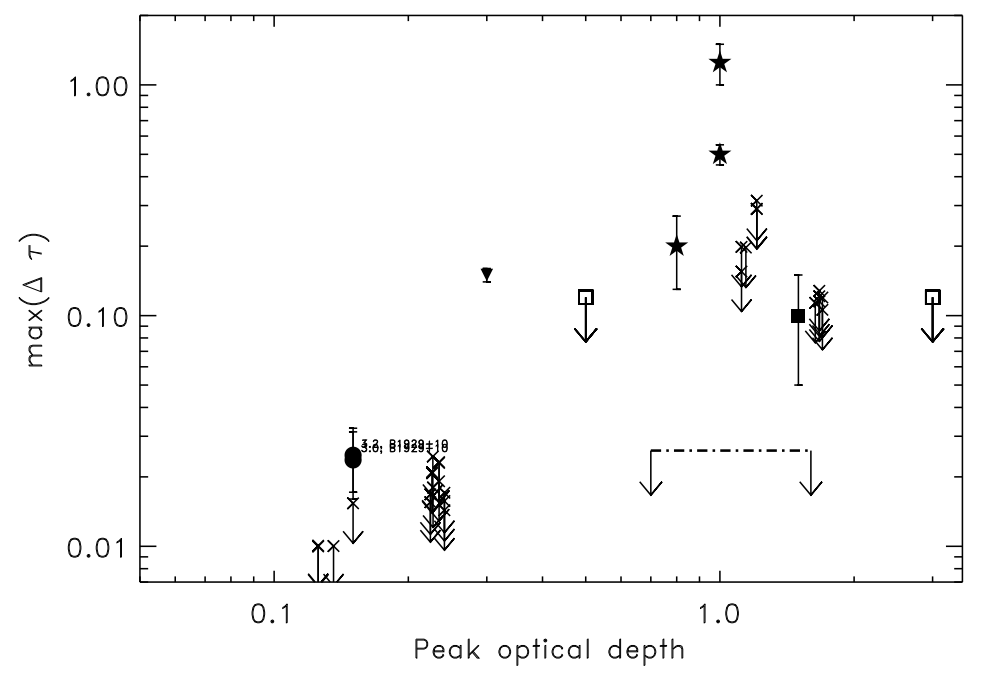

FIG. 12. - Variations in HI optical depth (and upper limits) as a function of peak optical depth along the line of sight. Circles (detections) and x's with arrows (upper limits) are from this study. Filled squares (detections) and open squares with arrows (non-detections) are from Johnston et al. (2003). The horizontal dot-dashed line anchored on either end by arrows represents non-detections over an almost continuous sampling of PSR $0329+54$ by Minter et al. (2005). The triangle at a peak optical depth of $\sim 0.3$ shows the 2.6- $\sigma$ detection in the direction of PSR B0301+19 by Weisberg et al. (2008). Interferometric detections are shown as stars: 3C138 at a peak optical depth of $\sim 0.8$ (Brogan et al. 2005); 3C161, the lower star at a peak optical depth of $\sim 1.0$ (Goss et al., 2008); and 3C147, the upper star at a peak optical depth of $\sim 1.0$ (Lazio et al. 2009). 


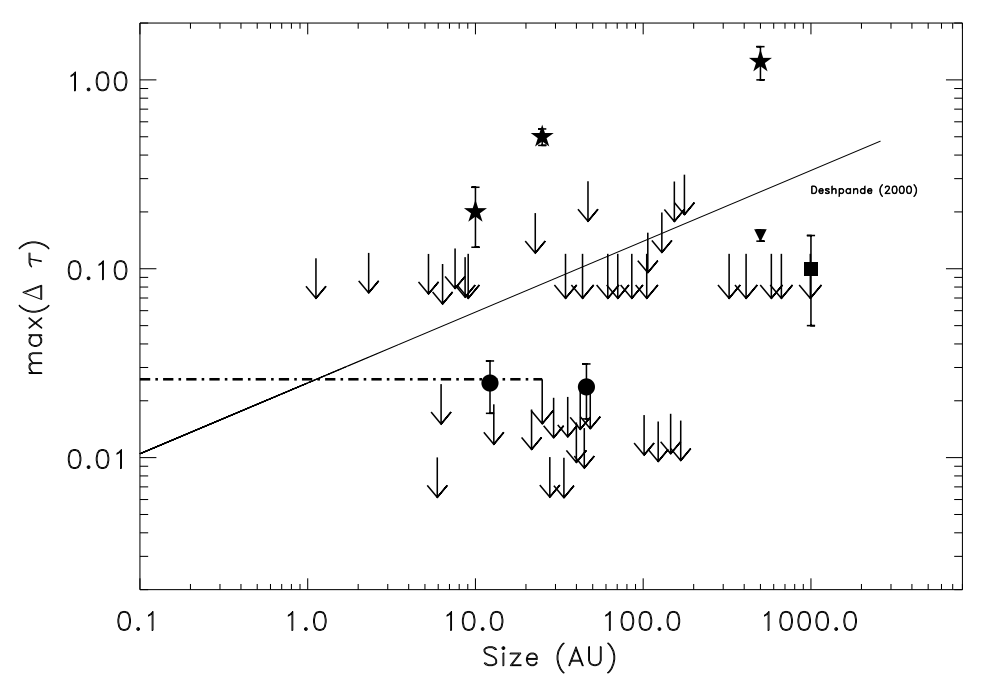

FIG. 13. - Variations in HI optical depth (and upper limits) as a function of spatial scale. Symbols are the same as in the previous figure. The interferometric detections (stars: 3C138 at 25 AU (Brogan et al. 2005); 3C161 at 500 AU (Goss et al.|2008); and 3C147 at 10 AU (Lazio et al. 2009). The solid line shows the level of fluctuations in $\Delta \tau$ as predicted by Deshpande (2000). The dot-dashed line represents an almost continuous sampling of PSR $0329+54$ by Minter et al. (2005) with no detections.

CNM turbulence corresponds to the viscous damping scale of $\sim 20 \mathrm{AU}$ (Zweibel 2005). However, in the case of magnetohydrodynamic (MHD) turbulence, the turbulent spectrum can cascade below the viscous cut-off scale down to the scale set by magnetic diffusion (Cho et al. 2002). In fact, magnetic and kinetic spectra can be greatly decoupled, with the magnetic spectrum being much shallower than the Kolmogorov spectrum.

\subsection{What's going on with TSAS?}

Our pulsar experiment shows only a few significant TSAS detections, all in the direction of one pulsar B1929+10. This result strongly suggests inhomogeneity in the galactic distribution of TSAS. We have examined two proposed origins of TSAS - (i) discrete HI blobs elongated along the line of sight; and (ii) fluctuations at the tail-end of the turbulent CNM spectrum; but our data do not provide support for either of these models. The principal problem is that we do not find these features to be homogeneously distributed. Meanwhile, recent numerical simulations do generate CNM clouds with a wide range of physical properties, including rare examples of very dense and over-pressured TSAS-like clouds; but the direct comparison between observations and simulated data is not straightforward since simulations probe much smaller ISM regions on scales somewhat larger than these experiments.

The most striking result from our examination of specific lines of sight is a possible correlation between the B1929+10 fluctuations and NaI interstellar clouds inside the Local Cavity or at the LB wall. As about one-half of B1929+10's line of sight is associated with the LB, and a significant fraction of this length appears to be traversing the LB wall, we suggest that the observed HI absorption fluctuations are most likely sampling small-scale structure in the LB. Numerous observations have indicated that the LB wall is highly inhomogeneous. Many interstellar cloudlets found inside the Local Cavity are thought to be caused by hydrodynamic instabilities fragmenting the LB wall. Through various studies, it has been shown that these cloudlets have velocities of up to $25 \mathrm{~km} \mathrm{~s}^{-1}$ relative to the Sun (Müller et al. 2006). This translates to $\sim 5 \mathrm{AU}$ $\mathrm{yr}^{-1}$. Coupled with the transverse speed of B1929+10 of $37 \mathrm{AU} \mathrm{yr}^{-1}$, this suggests that our observed TSAS could result from the pulsar's line of sight crossing very local cloudlets.

To investigate the origin of interstellar clouds inside the Local Cavity, we look to 2D hydrodynamical simulations of the WNM compression by supernova explosions and subsequent fragmentation into small clouds (Koyama \& Inutsuka 2002). They showed that within 0.3 Myr a thermally collapsing layer is formed behind the (supernova) shock front where cooling dominates heating and temperature monotonically decreases. As the gas cools it becomes thermally unstable, with a temperature in the range of 300-6000 K. Within $\sim 1 \mathrm{Myr}$ this layer starts to fragment into many small $\left(<10^{-2} \mathrm{pc}\right)$ cloudlets. Finally, these cloudlets can even reach thermal equilibrium at a temperature of $20 \mathrm{~K}$ and density of $2000 \mathrm{~cm}^{-3}$. As the ISM is frequently compressed by supernova explosions, fragmentation of the shock-compressed medium can provide a mechanism for the formation of many small cloudlets.

In the Koyama \& Inutsuka (2002) simulation, the cloudlets become embedded in the hot high-pressure gas as the shocked layer fragments, leading to a situation similar to what is observed in the LB. The exact temperature inside the Local Cavity has been uncertain (see Welsh et al. (2010b) for references), however it is clear that the Cavity is occupied by a warm/hot ionized medium. The cold cloudlets will eventually evaporate in the surrounding warm/hot medium. This process has been studied by many authors. The mass-loss rate in the case of classic evaporation (McKee \& Cowie 1977) is slightly lower when 
cooling is included (Nagashima et al. 2006). The mass-loss rate of Slavin (2007) who considered both flux saturation effects and radiative cooling is about 100 times lower than the classic evaporation rate. We continue by assuming the lowest suggested mass-loss rate, which will have the gentlest effect on the cold clouds.

The evaporation timescale depends greatly on the cloud size and the temperature of the surrounding medium. For a cloud size of $30 \mathrm{AU}, \tau_{\text {evap }}=10^{2}-10^{3} \mathrm{yr}$, depending upon whether the cloud is surrounded by the hot ionized medium (HIM) or WNM. For a larger cloud with a size of $10^{4} \mathrm{AU}, \tau_{\text {evap }}=10^{5-6}$ (HIM) or $10^{6-7}$ (WIM) yr. Clearly larger clouds survive longer and can even coalesce to build larger structures, even if surrounded by a hot medium. In about 1 Myr larger clouds could cross a large distance, $\sim 20 \mathrm{pc}$, and may even escape outside of the LB into the ISM through various chimneys and tunnels resulting from stellar winds and supernovae, and frequently observed in expanding shells. This scenario may be able to explain the existence of HI clouds on scales of thousands of AU or more in the ISM, as observed recently by Stanimirović \& Heiles (2005). On the contrary, very small clouds will evaporate quickly and therefore may not be common in the ISM. Their short inferred lifetimes indicate that they would usually be found close to their formation sites.

\section{SUMMARY}

We have used the Arecibo telescope to obtain multiepoch HI absorption spectra in the direction of six bright pulsars, with the goal of searching for structure on $\mathrm{AU}$ scales in the CNM. Our target sources were observed previously by Frail et al. (1994), who found pervasive tiny-scale HI structure. We used an advanced spectrometer capable of more accurate measurements in the face of wildly fluctuating pulsar signals, and carefully analyzed noise statistics. To search for variability of HI absorption profiles, which signifies inhomogeneities on AU scales in the CNM, we have searched for time-variability of the absorption line equivalent widths and of the absorption spectra themselves. While we had excellent sensitivity, significant variations in both EW and absorption spectra have been found only in the case of one pulsar: B1929+10. These variations imply TSAS on spatial scales $l=\frac{D_{\mathrm{HI}}}{350 \mathrm{pc}} \times(6,12,28,46) \mathrm{AU}$ (where $D_{\mathrm{HI}}$ is the distance to the TSAS feature), with an inferred volume density of $>10^{4} \mathrm{~cm}^{-3}$ and thermal pressure of $>10^{6} \mathrm{~cm}^{-3} \mathrm{~K}$.

Our study clearly shows the inhomogeneity in the galactic distribution of TSAS: the detection rate toward four of our pulsars is zero, while the detection rate toward B1929+10 is high. Such inhomogeneity is hard to explain with the model of discrete elongated disks or cylinders being a general property of CNM clouds (Heiles 1997); instead large spatial variations of TSAS physical properties or abundances must be invoked. While the Heiles (1997) model can help in bringing down the volume density and pressure of TSAS, the puzzles regarding its distribution and origin still remain.

Our examination of all pulsar and interferometric TSAS detections and upper limits does not show a correlation between the level of optical depth fluctuations and the TSAS spatial scale, as would be expected if the turbulent spectrum on much larger scales is extrapolated to AU-scales. The detections and non-detections probe an almost continuous range of spatial scales from $\sim 0.1$ to $1000 \mathrm{AU}$. Therefore, the large number of non-detections of TSAS suggests that the CNM clouds on scales $10^{-1}$ to $10^{3} \mathrm{AU}$ are not a pervasive property of the ISM. The sporadic TSAS detections on scales of tens of AU may indicate occasional local bursts of turbulent energy dissipation, rather than end-points of the turbulent spectrum.

Another striking result from our study is a possible correlation between B1929+10's TSAS and interstellar clouds observed in NaI absorption inside the Local Bubble. We find evidence that the TSAS is likely to be within $106 \mathrm{pc}$ of the Sun, and is sampling the small-scale structure of the LB caused by hydrodynamic instabilities fragmenting the LB wall. We propose that the line of sight of $\mathrm{B} 1929+10$ is revealing this recently formed small-scale structure. Similar bubbles and their walls are found throughout the Milky Way, but the lifetime of a TSAS cloud created from them depends strongly on the its size and the temperature of the surrounding medium. Larger fragments (size $\sim 10^{4}$ AU) survive longer and can travel large ISM distances, becoming a more general ISM property. On the other hand, the smallest clouds (size $\sim 10-100 \mathrm{AU}$ ) evaporate quickly close to their formation site, and are therefore not very commonly observed in the ISM. Additional processes, such as stellar mass-loss and collisions of interstellar clouds/filaments, probably contribute to the CNM structure formation on somewhat larger (sub-pc) scales.

We are grateful to Caltech's Center for Advance Computation and Research for the use of their facilities for data storage and processing. We express our thanks to Stuart Anderson, Rick Jenet, and Kathryn Devine for help with observations and data processing. We acknowledge the use of the ATNF Pulsar Catalog. We thank Carl Heiles, J.-P. Macquart, Miller Goss, Jay Gallagher, and Shu-ichiro Inutsuka for stimulating discussions. We would also like to thank the anonymous referee, whose comments improved greatly the clarity of the paper. SS acknowledges support from NSF grants AST-0097417 and AST-9981308, and the Research Corporation. She also thanks ASTRON and the Helena Kluyver visiting program for their hospitality during the final stage of the manuscript preparation. JMW, ZP, KT, and JTG were supported by NSF Grants AST0406832 and AST-0807556.

\section{REFERENCES}

Andrews, S. M., Meyer, D. M., \& Lauroesch, J. T. 2001, ApJ, 552, L73

Begum, A. \& et al. 2010, ApJ, in preparation

Bhat, N. D. R., Gupta, Y., \& Rao, A. P. 1998, ApJ, 500, 262

Braun, R. \& Kanekar, N. 2005, A\&A, 436, 53

Breitschwerdt, D. \& de Avillez, M. A. 2006, A\&A, 452, L1
Brisken, W. F., Benson, J. M., Goss, W. M., \& Thorsett, S. E. 2002, ApJ, 571, 906

Brisken, W. F., Fruchter, A. S., Goss, W. M., Herrnstein, R. M., \& Thorsett, S. E. 2003, AJ, 126, 3090 
Brogan, C. L., Goss, W. M., Lazio, T. J. W., \& Faison, M. D. 2007, in Astronomical Society of the Pacific Conference Series, Vol. 365, SINS - Small Ionized and Neutral Structures in the Diffuse Interstellar Medium, ed. M. Haverkorn \& W. M. Goss, 12

Brogan, C. L., Zauderer, B. A., Lazio, T. J., Goss, W. M., DePree, C. G., \& Faison, M. D. 2005, AJ, 130, 698

Burton, W. B. \& Hartmann, D. 1994, Ap\&SS, 217, 189

Chatterjee, S., Cordes, J. M., Lazio, T. J. W., Goss, W. M., Fomalont, E. B., \& Benson, J. M. 2001, ApJ, 550, 287

Chatterjee, S., Cordes, J. M., Vlemmings, W. H. T., Arzoumanian, Z., Goss, W. M., \& Lazio, T. J. W. 2004, ApJ, 604, 339

Cho, J., Lazarian, A., \& Vishniac, E. T. 2002, ApJ, 566, L49

Clifton, T. R., Frail, D. A., Kulkarni, S. R., \& Weisberg, J. M. 1988, ApJ, 333, 332

Crawford, I. A., Howarth, I. D., Ryder, S. D., \& Stathakis, R. A. 2000, MNRAS, 319, L1

Davis, R. J., Diamond, P. J., \& Goss, W. M. 1996, MNRAS, 283, 1105

de Geus, E. J., Bronfman, L., \& Thaddeus, P. 1990, A\&A, 231, 137

Deshpande, A. A. 2000, MNRAS, 317, 199

Deshpande, A. A., Dwarakanath, K. S., \& Goss, W. M. 2000, ApJ, 543, 227

Deshpande, A. A., McCulloch, P. M., Radhakrishnan, V., \& Anantharamaiah, K. R. 1992, MNRAS, 258, 19P

Diamond, P. J., Goss, W. M., Romney, J. D., Booth, R. S., Karbela, P. N. M., \& Mebold, U. 1989, ApJ, 347, 302

Dickey, J. M. \& Lockman, F. J. 1990, ARA\&A, 28, 215

Dickey, J. M., McClure-Griffiths, N. M., Gaensler, B. M., \& Green, A. J. 2003, ApJ, 585, 801

Dieter, N. H., Welch, W. J., \& Romney, J. D. 1976, ApJ, 206, L113

Elmegreen, B. G. \& Scalo, J. 2004, ARA\&A, 42, 211

Faison, M. D. \& Goss, W. M. 2001, AJ, 121, 2706

Faison, M. D., Goss, W. M., Diamond, P. J., \& Taylor, G. B. 1998, AJ, $116,2916 \mathrm{~F}$

Frail, D. A., Weisberg, J. M., Cordes, J. M., \& Mathers, C. 1994, ApJ, 436, 144

Genova, R., Beckman, J. E., Bowyer, S., \& Spicer, T. 1997, ApJ, 484,761

Goss, W. M., Richards, A. M. S., Muxlow, T. W. B., \& Thomasson, P. 2008, ArXiv e-prints, 804

Gupta, Y., Bhat, N. D. R., \& Rao, A. P. 1999, ApJ, 520, 173

Gwinn, C. R. 2001, ApJ, 561, 815

Gwinn, C. R., Reynolds, J. E., \& Wilson, W. W. 2007, in Astronomical Society of the Pacific Conference Series, Vol. 365, SINS - Small Ionized and Neutral Structures in the Diffuse Interstellar Medium, ed. M. Haverkorn \& W. M. Goss, 90

Gwinn, C. R., Taylor, J. H., Weisberg, J. M., \& Rawley, L. A. 1986, AJ, 91,338

Harrison, P. A., Lyne, A. G., \& Anderson, B. 1993, MNRAS, 261, 113

Haslam, C. G. T., Stoffel, H., Salter, C. J., \& Wilson, W. E. 1982, A\&AS, 47, 1

Haverkorn, M. \& Goss, W. M., eds. 2007, Astronomical Society of the Pacific Conference Series, Vol. 365, SINS - Small Ionized and Neutral Structures in the Diffuse Interstellar Medium

Heiles, C. 1997, ApJ, 481, 193

Heiles, C., et al. 2001, PASP, 113, 1247

Heiles, C. 2007a, PASP, 119, 643

Heiles, C. 2007b, in Astronomical Society of the Pacific Conference Series, Vol. 365, SINS - Small Ionized and Neutral Structures in the Diffuse Interstellar Medium, ed. M. Haverkorn \& W. M. Goss, 3
Heiles, C. \& Troland, T. H. 2003a, ApJS, 145, 329

-. 2003b, ApJ, 586, 1067

Hennebelle, P. \& Audit, E. 2007, A\&A, 465, 431

Hennebelle, P., Audit, E., \& Miville-Deschênes, M.-A. 2007, A\&A, 465,445

Isobe, T., Feigelson, E. D., \& Nelson, P. I. 1986, ApJ, 306, 490

Jenet, F. A., Cook, W. R., Prince, T. A., \& Unwin, S. C. 1997, PASP, 109, 707

Jenkins, E. B. \& Tripp, T. M. 2001, ApJS, 137, 297

Johnston, S., Koribalski, B., Wilson, W., \& Walker, M. 2003, MNRAS, 341, 941

Kanekar, N. \& Chengalur, J. N. 2001, MNRAS, 325, 631

Koyama, H. \& Inutsuka, S. 2002, ApJ, 564, L97

Lallement, R., Welsh, B. Y., Vergely, J. L., Crifo, F., \& Sfeir, D. 2003, A\&A, 411, 447

Lauroesch, J. T. \& Meyer, D. M. 2003, ApJ, 591, L123

Lazio, T. J. W., Brogan, C. L., Goss, W. M., \& Stanimirović, S. 2009, AJ, 137, 4526

Mac Low, M.-M., Balsara, D. S., Kim, J., \& de Avillez, M. A. 2005, ApJ, 626, 864

Manchester, R. N., Hobbs, G. B., Teoh, A., \& Hobbs, M. 2005, AJ, 129, 1993

McKee, C. F. \& Cowie, L. L. 1977, ApJ, 215, 213

Meyer, D. M. \& Blades, J. C. 1996, ApJ, 464, L179

Minter, A. H., Balser, D. S., \& Kartaltepe, J. S. 2005, ApJ, 631, 376

Müller, H.-R., Frisch, P. C., Florinski, V., \& Zank, G. P. 2006, ApJ, 647,1491

Nagashima, M., Inutsuka, S., \& Koyama, H. 2006, ApJ, 652, L41

Phillips, J. A. \& Clegg, A. W. 1992, Nature, 360, 137

Rohlfs, K., \& Wilson, T. L. 2004, Tools of radio astronomy, 4th ed. Berlin: Springer, 2004, 107

Roy, N., Chengalur, J. N., Dutta, P., \& Bharadwaj, S. 2010, MNRAS, $\mathrm{L} 33+$

Scalo, J. \& Elmegreen, B. G. 2004, ARA\&A, 42, 275

Slavin, J. D. 2007, in Astronomical Society of the Pacific Conference Series, Vol. 365, SINS - Small Ionized and Neutral Structures in the Diffuse Interstellar Medium, ed. M. Haverkorn \& W. M. Goss, 113

Spitzer, L. J. 1978, Physical processes in the interstellar medium (New York, USA: J. Wiley \& Sons, Inc), 1

Stanimirović, S. \& Heiles, C. 2005, ApJ, 631, 371

Stanimirović, S., Heiles, C., \& Kanekar, N. 2007, in Astronomical Society of the Pacific Conference Series, Vol. 365, SINS - Small Ionized and Neutral Structures in the Diffuse Interstellar Medium, ed. M. Haverkorn \& W. M. Goss, 22

Stanimirović, S., Weisberg, J. M., Hedden, A., Devine, K. E., \& Green, J. T. 2003, ApJ, 598, L23

Toscano, M., Britton, M. C.. Manchester, R. N., Bailes, M., Sandhu, J. S., Kulkarni, S. R., \& Anderson, S. B. 1999, ApJ, 523, L171

Weisberg, J. M., Rankin, J., \& Boriakoff, V. 1980, A\&A, 88, 84

Weisberg, J. M. \& Stanimirović, S. 2007, in Astronomical Society of the Pacific Conference Series, Vol. 365, SINS - Small Ionized and Neutral Structures in the Diffuse Interstellar Medium, ed. M. Haverkorn \& W. M. Goss, 28

Weisberg, J. M., Stanimirović, S., Xilouris, K., Hedden, A., de la Fuente, A., Anderson, S. B., \& Jenet, F. A. 2008, ApJ, 674, 286

Welsh, B. Y., Lallement, R., Vergely, J., \& Raimond, S. 2010a, A\&A, 510, A5 4

Welsh, B. Y., Wheatley, J., Siegmund, O. H. W., \& Lallement, R. 2010b, ApJ, 712, L199

Zweibel, E. 2005, in SINS - Small Ionized and Neutral Structures in the Diffuse Interstellar Medium; http://www.astro.wisc.edu/ sstanimi/Tiny/SINS_Talks1/zweibel1.pdf 